\title{
THE COEFFICIENT PROBLEM FOR SCHLICHT MAPPINGS OF THE EXTERIOR OF THE UNIT CIRCLE
}

\author{
BY \\ G. SPRINGER
}

1. Let $T$ represent the class of analytic functions $f$ which are regular and schlicht in $|z|>1$ with the exception of a simple pole at infinity where they have the normalized Laurent expansion

$$
w=f(z)=z+\frac{a_{1}}{z}+\frac{a_{2}}{z^{2}}+\frac{a_{3}}{z^{3}}+\cdots .
$$

Furthermore, let $S$ denote the class of those functions $\phi$ which are inverses of functions in $T$; hence $\phi$ affords a conformal map of a domain $D$ (of mapping radius one) containing $w=\infty$ onto the exterior of the unit circle $C:|z|>1$ and at $w=\infty, \phi$ has the normalization

$$
\phi(w)=w+\frac{b_{1}}{w}+\frac{b_{2}}{w^{2}}+\frac{b_{3}}{w^{3}}+\cdots .
$$

The underlying problem confronting us in this paper is the determination of precise upper bounds for the moduli of the coefficients in these normalized expansions at $\infty$ for functions in the classes $S$ and $T$. This problem for the first two coefficients in each class has already been solved. We note first that since $\phi$ and $f$ are inverse, $a_{1}=-b_{1}$ and $a_{2}=-b_{2}$, so for these two cases, discussion of only one of the two classes is necessary. For higher coefficients, the $a$ 's and $b$ 's are related by certain polynomials, so in general, a separate study must be made for each class.

Gronwall [6](1), Bieberbach [1], and Faber [3] showed that $\left|a_{1}\right| \leqq 1$ with the unique function

$$
f(z)=z+e^{i \alpha} / z, \quad \alpha \text { real, }
$$

giving us the equality. This function maps the exterior of the unit circle onto the entire $w$-plane with the exception of a cut along a line segment of length 4 which is bisected by the point $w=0$. In 1938, Golusin [5] and Schiffer [13] proved that $\left|a_{2}\right| \leqq 2 / 3$, with equality holding only for the function

$$
f(z)=z\left(1+\frac{e^{i \alpha}}{z^{3}}\right)^{2 / 3}, \quad \alpha \text { real }
$$

Presented to the International Congress of Mathematicians, September 4, 1950; received by the editors June 5,1950 .

(1) Numbers in brackets refer to the bibliography at the end of the paper. 
which maps $|z|>1$ onto the whole $w$-plane except for a cut consisting of three lines, each of length $2^{2 / 3}$, radiating symmetrically from the origin.

In this paper, the precise upper bound for $\left|b_{3}\right|$ is established. Furthermore, certain general properties of the extremal functions for the problem of maximizing $\left|b_{n}\right|$ are found. Certain general facts are found concerning the region of variability of the points $\left(a_{1}, a_{2}, \cdots, a_{n}\right)$ in the space of $2 n$ real dimensions.

2. In this study, variational methods are applied to the mapping functions in the classes $T$ and $S$ from which conditions are derived for the extremal functions. The point of departure will be the Julia [8] variation formula for the mapping functions, which is a specialization of the Hadamard [7] variation formula for the Green's function. Because of its central role in the subsequent investigation, a derivation of this formula will be briefly sketched which is different and simpler than those in the literature.

Let $D$ represent a simply connected domain in the $w$-plane, bounded by an analytic curve and containing the point at infinity. We shall denote by $f(z)$ a schlicht function defined in the exterior of the unit circle, $C:|z|>1$, and mapping $C$ onto $D$ such that the points at infinity correspond. The inverse of $f(z)$ will be called $\phi(w)$. At the outset, we shall ask that $f(z)$ be normalized so that in the neighborhood of infinity,

$$
w=f(z)=a z+a_{0}+\frac{a_{1}}{z}+\frac{a_{2}}{z^{2}}+\cdots, \quad a>0 .
$$

Let us now make a small variation in the domain $D$, calling the new domain $D^{*}$. $D^{*}$ will also be assumed to be bounded by an analytic curve. We may consider the mapping $f^{*}(z)$ taking $C$ onto $D^{*}$ which has the following normalization at infinity:

$$
f^{*}(z)=a^{*} z+a_{0}^{*}+\frac{a_{1}^{*}}{z}+\frac{a_{2}^{*}}{z^{2}}+\cdots, \quad a^{*}>0 .
$$

Using the conventional variational notation $\delta f(z)=f^{*}(z)-f(z)$, the function $\delta f(z) / z f^{\prime}(z)$ is regular in $C$. Hence its real part is harmonic and may be represented by the Poisson integral:

$$
\operatorname{Re} \frac{\delta f(x)}{x f^{\prime}(x)}=\frac{1}{2 \pi} \oint_{\gamma} \operatorname{Re}\left(\frac{z+x}{z-x}\right) \operatorname{Re}\left(\frac{\delta f(z)}{z f^{\prime}(z)}\right) \frac{d z}{i z},
$$

where $x$ is an interior point of $C$, the integration extending over the boundary $\gamma$ of $C$ and taken in a positive direction relative to $C$ (clockwise around $\gamma$ ).

Let $s$ be the parameter of arc length on the boundary $\Gamma$ of $D$, chosen in such a way that $s$ increases when $\Gamma$ is traversed in the positive sense. Since

$$
d w=f^{\prime}(z) d z=i z f^{\prime}(z) d \phi
$$


when $z=e^{i \phi}$, we may write

$$
\operatorname{Re}\left[\frac{\delta f(z)}{z f^{\prime}(z)}\right] \frac{d z}{i z}=\operatorname{Re}\left[\frac{\delta f(z)}{-i w^{\prime}}\right] \frac{d \phi}{d s} d \phi
$$

in which $w^{\prime}=d w / d s$ is a unit vector directed along the tangent to $\Gamma$. Then $i w^{\prime}$ is a unit vector directed along the interior normal to $\Gamma . \operatorname{Re}\left[\delta f(z) / i w^{\prime}\right]=\delta n$ is the length of the projection of the shift $\delta f(z)$ in the direction of the normal to $\Gamma$, taken to be positive toward the interior of $D$. Thus (7) may be rewritten

$$
\begin{aligned}
\operatorname{Re} \frac{\delta f(x)}{x f^{\prime}(x)} & =-\frac{1}{2 \pi} \oint_{\gamma} \operatorname{Re}\left(\frac{z+x}{z-x}\right) \delta n \frac{d \phi}{d s} d \phi \\
& =\frac{1}{2 \pi} \oint_{\gamma} \operatorname{Re}\left(\frac{z+x}{z-x}\right)\left[i w^{\prime} \delta n\right] \frac{d w}{i z^{2} f^{\prime}(z)^{2}} .
\end{aligned}
$$

We now observe that $\delta w=i w^{\prime} \delta n$ is the normal component of the displacement $\delta f(z)$; this leads to the formula:

$$
\operatorname{Re} \frac{\delta f(x)}{x f^{\prime}(x)}=\frac{1}{2 \pi} \oint_{\gamma} \operatorname{Re}\left(\frac{z+x}{z-x}\right) \frac{\delta w d w}{i z^{2} f^{\prime}(z)^{2}} .
$$

The term

$$
\frac{\delta w d w}{i z^{2} f^{\prime}(z)^{2}}=-\delta n \frac{d \phi}{d s} d \phi
$$

is real on $\gamma$, so we may write

$$
\operatorname{Re} \frac{\delta f(x)}{x f^{\prime}(x)}=\operatorname{Re} \frac{1}{2 \pi} \oint_{\gamma} \frac{z+x}{z-x} \frac{\delta w d w}{i z^{2} f^{\prime}(z)^{2}},
$$

in which the integral on the right is an analytic function of $x$. Consequently we may complete these harmonic functions to analytic functions; namely,

$$
\frac{\delta f(x)}{x f^{\prime}(x)}=\frac{1}{2 \pi} \oint_{\gamma} \frac{z+x}{z-x} \frac{\delta w d w}{i z^{2} f^{\prime}(z)^{2}}+i k, \quad k=\text { real constant } .
$$

To evaluate $k$, let $x$ become infinite, then

$$
\frac{\delta a}{a}=\frac{a^{*}-a}{a}=-\frac{1}{2 \pi} \oint_{\gamma} \frac{\delta w d w}{i z^{2} f^{\prime}(z)^{2}}+i k .
$$

Since the term on the left and the integral on the right are both real, $k=0$. Thus

$$
\delta f(x)=x f^{\prime}(x) \frac{1}{2 \pi} \oint_{\gamma} \frac{z+x}{z-x} \frac{\delta w d w}{i z^{2} f^{\prime}(z)^{2}}
$$


In this derivation, $\delta w$ has meant the normal component of the displacement $\delta f$; that is, $\delta w=i w^{\prime} \operatorname{Re}\left(\delta f / i w^{\prime}\right)$. In practice, however, the boundaries $\Gamma$ and $\Gamma^{*}$ are given, and, in general, the function $f^{*}$ will not be known, making it difficult to determine $\delta f(z)$ or the normal component of $\delta f(z)$. It will be more convenient to work with the actual normal displacement; that is, the vector obtained by going from $\Gamma$ to $\Gamma^{*}$ along the normal to $\Gamma$.

It can be shown without difficulty that if the actual normal displacement is regular enough, say it is a twice continuously differentiable function of the arc length $s$, and if $\Gamma$ and $\Gamma^{*}$ are in a Fréchet neighborhood of order $\epsilon$ (that is, to each point $w$ of $\Gamma$ we can associate a point $w^{*}$ of $\Gamma^{*}$ such that $\left|w-w^{*}\right|$ $=O(\epsilon)$ and vice versa), we can interpret the $\delta w$ in (16) as the actual normal displacement and introduce an error of at most order $\epsilon^{2}$ in the expression for $\delta f(x)$. These details will not be presented here; formula (15) with $\delta n$ interpreted as the actual normal displacement is the variation formula of Julia in a form given by Biernacki [2].

The class of functions $f(z)$ in which we make the variation will now be restricted by a further normalization at infinity; namely, we shall require that in the neighborhood of infinity,

$$
f(z)=z+a_{1} / z+a_{2} / z^{2}+\cdots .
$$

If $f(z)=g(z) / a$ and $f^{*}(z)=g^{*}(z) / a^{*}$, then

$$
\delta f(z)=\delta g(z) / a-g(z) \delta a / a^{2},
$$

again considering only first order terms (neglecting terms $\boldsymbol{o}(\boldsymbol{\epsilon})$ ). Applying formula (16) to $g(z)$ and taking $\delta a$ from (15) with $k=0$, we get

$$
\delta f(x)=x f^{\prime}(x) \oint_{\gamma} \frac{z+x}{z-x} \frac{1}{2 \pi i} \frac{\delta w d w}{z^{2} f^{\prime}(z)^{2}}+f(x) \oint_{\gamma} \frac{1}{2 \pi i} \frac{\delta w d w}{z^{2} f^{\prime}(z)^{2}} .
$$

The added term restricts the variation to the class of functions having derivative one at infinity, but has not yet restricted the variation so as to leave the constant term zero. To do this, we expand the right-hand side in powers of $x$ and subtract the constant term; for if $f(x)=g(x)-a_{0}$ and $f^{*}(x)$ $=g^{*}(x)-a_{0}^{*}$, we have $\delta f(x)=\delta g(x)-\delta a_{0}$. This leads to the final variation formula:

$$
\delta f(x)=x f^{\prime}(x) \oint_{\gamma} \frac{z+x}{z-x} \frac{1}{2 \pi i} \frac{\delta w d w}{z^{2} f^{\prime}(z)^{2}}+\oint_{\gamma}(f(x)+2 z) \frac{1}{2 \pi i} \frac{\delta w d w}{z^{2} f^{\prime}(z)^{2}} .
$$

Let $z=\phi(w)$ be the inverse of $w=f(z)$; then $\phi(w)$ has a power series expansion at infinity of the form

$$
\phi(w)=w+b_{1} / w+b_{2} / w^{2}+\cdots .
$$

We also let $\phi^{*}(w)$ be the inverse of $f^{*}(z)$; then we can set $y^{*}=f^{*}(x)$ and $y=f(x)$. The variation of $\phi(y)$ can be found by noting that $x=\phi^{*}\left(y^{*}\right)=\phi(y)$; 
thus

$$
\begin{aligned}
\delta \phi(y) & =\phi^{*}(y)-\phi(y)=\phi^{*}(y)-\phi^{*}\left(y^{*}\right) \\
& =-\phi^{* \prime}(y)\left(y^{*}-y\right)+O\left(\epsilon^{2}\right) \\
& =-\phi^{* \prime}(y) \delta f(x)+O\left(\epsilon^{2}\right),
\end{aligned}
$$$$
\left|y^{*}-y\right|=O(\epsilon) \text {. }
$$

However, $\phi^{* \prime}(y)=\phi^{\prime}(y)+O(\epsilon)$, so for a first variation,

$$
\delta \phi(y)=-\phi^{\prime}(y) \delta f(x) .
$$

Substituting (23) into (20) gives the following variation formula for the normalized inverse functions $z=\phi(w), x=\phi(y)$ :

$$
\begin{aligned}
\delta \phi(y)= & -\phi(y) \oint_{\Gamma} \frac{\phi(w)+\phi(y)}{\phi(w)-\phi(y)} \frac{1}{2 \pi i} \frac{\phi^{\prime}(w)^{2}}{\phi(w)^{2}} \delta w d w \\
& -\oint_{\Gamma}\left[y \phi^{\prime}(y)+2 \phi(w) \phi^{\prime}(y)\right] \frac{1}{2 \pi i} \frac{\phi^{\prime}(w)^{2}}{\phi(w)^{2}} \delta w d w .
\end{aligned}
$$

Since we are interested in studying the extremal coefficients of $\phi(w)$, let us obtain the variation formula for the coefficient $b_{n}$ by expanding both sides of (24), in powers of $y$, and comparing coefficients. We first note that (24) can be written in the form:

$$
\begin{aligned}
\delta \phi(y)=\oint_{\Gamma}\left[\frac{2 \phi(w)^{2}}{\phi(y)-\phi(w)}+2 \phi(w)+\right. & \phi(y)-y \phi^{\prime}(y) \\
& \left.-2 \phi(w) \phi^{\prime}(y)\right] \frac{1}{2 \pi i} \frac{\phi^{\prime}(w)^{2}}{\phi(w)^{2}} \delta w d w .
\end{aligned}
$$

The term

$$
\frac{1}{\phi(y)-t}=\sum_{n=1}^{\infty} \frac{1}{n} P_{n}^{\prime}(t) y^{-n}
$$

is the generating function for the derivatives of the Faber polynomials $P_{n}(t)$ of degree $n$ (Schiffer [14]). We then obtain

$$
\begin{aligned}
\delta b_{n}=\oint_{\Gamma}\left[\frac{2}{n} \phi(w)^{2} P_{n}^{\prime}(\phi(w))+(n\right. & +1) b_{n} \\
& \left.+2 \phi(w)(n-1) b_{n-1}\right] \frac{1}{2 \pi i} \frac{\phi^{\prime}(w)^{2}}{\phi(w)^{2}} \delta w d w,
\end{aligned}
$$

or

$$
\delta b_{n}=\oint_{\gamma}\left[\frac{2}{n} z^{2} P_{n}^{\prime}(z)+(n+1) b_{n}+2 z(n-1) b_{n-1}\right] \frac{1}{2 \pi i} \frac{\delta w d w}{z^{2} f^{\prime}(z)^{2}} .
$$


The variation formulas (20), (25), and (28) were derived under the assumption that the boundary $\Gamma$ was analytic. Since we have no guarantee that our extremals will haye sufficiently smooth boundaries, we must try to eliminate this restriction by removing the integration over the boundary. This can be done by specializing the variation of $\Gamma$ to the following:

$$
w^{*}=w+\frac{a \rho^{2}}{w-w_{0}},
$$

where $w_{0}$ is an arbitrary fixed point in $D, \rho$ is a positive number, and $|a|=1$. The function $w^{*}(w)$ is univalent in the exterior of a circle of radius $\rho$ about $w_{0}$; and hence affords a one-to-one mapping of $\Gamma$ onto an analytic curve $\Gamma^{*}$ if $\rho$ is sufficiently small. The normal projection of this shift of $\Gamma$ is

$$
\delta w=i w^{\prime} \operatorname{Re}\left(\frac{1}{i w^{\prime}} \frac{a \rho^{2}}{w-w_{0}}\right) \frac{w^{\prime} d w}{z^{2} f^{\prime}(z)^{2}},
$$

and substitution into (16) yields

$$
\begin{aligned}
\frac{\delta f(x)}{x f^{\prime}(x)}= & \frac{1}{2 \pi} \oint_{\gamma} \frac{z+x}{z-x} \operatorname{Re}\left(\frac{1}{i w^{\prime}} \frac{a \rho^{2}}{w-w_{0}}\right) \frac{w^{\prime} d w}{z^{2} f^{\prime}(z)^{2}} \\
= & \frac{1}{4 \pi i} \oint_{\gamma} \frac{z+x}{z-x} \frac{a \rho^{2}}{w-w_{0}} \frac{d w}{z^{2} f^{\prime}(z)^{2}} \\
& +\frac{1}{4 \pi i} \oint_{\gamma} \frac{1+\bar{x} z}{1-\bar{x} z} \frac{a \rho^{2}}{w-w_{0}} \frac{d w}{z^{2} f^{\prime}(z)^{2}}
\end{aligned}
$$

since $\left(i w^{\prime} d w\right) / z^{2} f^{\prime}(z)^{2}$ is real on $\gamma$, and $\bar{z}=1 / z$ on $\gamma$. We can apply the residue theorem to these integrals to obtain, with $w_{0}=f\left(z_{0}\right)$,

$$
\begin{aligned}
\delta f(x)= & a \rho^{2} \frac{1}{f(x)-f\left(z_{0}\right)}+\frac{a \rho^{2}}{2} \frac{z_{0}+x}{z_{0}-x} \frac{x f^{\prime}(x)}{z_{0}^{2} f^{\prime}\left(z_{0}\right)^{2}} \\
& +\frac{\bar{a} \rho^{2}}{2} \frac{1+\bar{z}_{0} x}{1-\bar{z}_{0} x} \frac{x f^{\prime}(x)}{\bar{z}_{0}^{2} \bar{f}^{\prime}\left(z_{0}\right)^{2}} .
\end{aligned}
$$

This variation is not yet normalized to remain in the class $T$. Going through the same procedure used in normalizing (16) to get (20), we obtain the normalized variation formula

$$
\begin{aligned}
\delta f(x)= & \frac{a \rho^{2}}{2}\left[\frac{2}{f(x)-f\left(z_{0}\right)}+\left(\frac{z_{0}+x}{z_{0}-x} x f^{\prime}(x)+f(x)+2 z_{0}\right) \frac{1}{z_{0}^{2} f^{\prime}\left(z_{0}\right)^{2}}\right. \\
& +\frac{\bar{a} \rho^{2}}{2}\left[\left(\frac{1+\bar{z}_{0} x}{1-\bar{z}_{0} x} x f^{\prime}(x)+f(x)+\frac{2}{\bar{z}_{0}}\right) \frac{1}{\bar{z}_{0}^{2} \bar{f}^{\prime}\left(z_{0}\right)^{2}} \cdot\right.
\end{aligned}
$$

Using (23), we obtain the corresponding variation formula for $\phi(w)$; namely, 
$\delta \phi(y)=-\frac{a \rho^{2}}{2}\left[\frac{2 \phi^{\prime}(y)}{w-w_{0}}+\left(\frac{\phi\left(w_{0}\right)+\phi(y)}{\phi\left(w_{0}\right)-\phi(y)} \phi(y)+y \phi^{\prime}(y)\right.\right.$

$$
\begin{array}{r}
\left.\left.+2 \phi\left(w_{0}\right) \phi^{\prime}(y)\right) \frac{\phi^{\prime}\left(w_{0}\right)^{2}}{\phi\left(w_{0}\right)^{2}}\right] \\
-\frac{\bar{a} \rho^{2}}{2}\left[\frac{1+\phi(y) \Phi\left(w_{0}\right)}{1-\phi(y) \Phi\left(w_{0}\right)} \phi(y)+y \phi^{\prime}(y)+\frac{2 \phi^{\prime}(y)}{\Phi\left(w_{0}\right)}\right] \frac{\phi^{\prime}\left(w_{0}\right)^{2}}{\Phi\left(w_{0}\right)^{2}}
\end{array}
$$

where $w_{0}=f\left(z_{0}\right)$.

Expanding both sides of (34) in powers of $y$, we obtain the variation formulas for the coefficients $b_{n}$ :

$$
\begin{aligned}
\delta b_{n}= & \frac{a \rho^{2}}{2}\left\{\left[\frac{2}{n} \phi\left(w_{0}\right)^{2} P_{n}^{\prime}\left(\phi\left(w_{0}\right)\right)+(n+1) b_{n+1}+2 \phi\left(w_{0}\right)(n-1) b_{n-1}\right] \frac{\phi^{\prime}\left(w_{0}\right)^{2}}{\phi\left(w_{0}\right)^{2}}\right. \\
& \left.+2 \sum_{\nu=0}^{n-1}(n-\nu-2) b_{n-\nu-2} w_{0}^{\nu}\right\} \\
& +\frac{\frac{a \rho^{2}}{2}\left[\frac{2}{n} \frac{1}{\phi\left(w_{0}\right)^{2}} \bar{P}_{n}^{\prime}\left(\frac{1}{\phi\left(w_{0}\right)}\right)+(n+1) b_{n}+\frac{2}{\phi\left(w_{0}\right)}(n-1) b_{n-1}\right] \frac{\phi^{\prime}\left(w_{0}\right)^{2}}{\phi\left(w_{0}\right)^{2}}}{}
\end{aligned}
$$

where $b_{-1}=1$ and $b_{0}=0$, the $\bar{P}_{n}(t)$ representing the polynomial whose coefficients are the complex conjugates of those of $P_{n}(t)$. In terms of $z$, this becomes

$$
\delta b_{n}=\frac{a \rho^{2}}{2}\left\{\left[\frac{2}{n} z_{0}^{2} P_{n}^{\prime}\left(z_{0}\right)+(n+1) b_{n}+2 z_{0}(n-1) b_{n-1}\right] \frac{1}{z_{0}^{2} f^{\prime}\left(z_{0}\right)^{2}}\right.
$$

$$
+\frac{\left.2 \sum_{\nu=0}^{n-1}(n-\nu-2) b_{n-\nu-2} f\left(z_{0}\right)^{\nu}\right\}}{+\frac{a \rho^{2}}{2}\left[\frac{2}{n} \frac{1}{z_{0}^{2}} \bar{P}_{n}^{\prime}\left(\frac{1}{z_{0}}\right)+(n+1) b_{n}+\frac{2}{z_{0}}(n-1) b_{n-1}\right] \frac{1}{z_{0}^{2} f^{\prime}\left(z_{0}\right)^{2}} .}
$$

The corresponding variation formula for the coefficients $a_{n}$ can be found by expanding (33) in powers of $x$ :

$$
\begin{aligned}
\delta a_{n}= & \frac{a \rho^{2}}{2}\left\{\left[(n+1) a_{n}+2 \sum_{\nu=1}^{n+1}(n-\nu) a_{n-\nu} z_{0}^{\nu}\right] \frac{1}{z_{0}^{2} f^{\prime}\left(z_{0}\right)^{2}}+\frac{2}{n} R_{n}^{\prime}\left(f\left(z_{0}\right)\right)\right\} \\
& +\frac{a \rho^{2}}{2}\left[(n+1) a_{n}+2 \sum_{\nu=1}^{n+1}(n-\nu) \bar{a}_{n-\nu} \frac{1}{z_{0}^{\nu}}\right] \frac{1}{z_{0}^{2} f^{\prime}\left(z_{0}\right)^{2}},
\end{aligned}
$$

$R_{n}(t)$ being the Faber polynomial of degree $n$ generated by

$$
\frac{1}{f(z)-t}=\sum_{n=1}^{\infty} \frac{1}{n} R_{n}^{\prime}(t) z^{-n} \text {. }
$$


The variation formulas (33) to (37) no longer involve contour integrals explicitly, but are "interior" variations in the sense that they appear to be independent of the boundary behavior of the functions. Since we initially made the assumption that $\Gamma$ was analytic, it would appear that this restriction must also be placed on formulas (33) to (37). An arbitrary boundary curve may, however, be considered as the limit of a sequence of analytic curves (Walsh [17]). The above formulas are valid for the approximating analytic curves, and since they involve only the mapping function and its derivatives at the given interior point, (33) to (37) converge uniformly to the desired variation formulas for arbitrary domains.

In the preceding limiting operation, however, we have neglected an important consideration; that is, we have not shown that the terms of higher order, which we neglected in considering only the first variation, also converge uniformly and do not affect the first variation. To demonstrate this, we shall use the knowledge of the form of the variation formulas (32) and (34) obtained for analytic boundaries from the Julia formula to motivate a direct proof of the same formulas, which, however, will allow us to make a better estimate of the higher order terms when we extend to nonanalytic boundaries. (See Garabedian and Schiffer [4].) This will later enable us to conclude that the extremal problems we consider are solved by functions mapping the circle onto domains bounded by analytic arcs. Thus the original forms of the Julia formulas (20), (25), and (28) can be applied and will lead to several important results in characterizing the extremal domains beyond the analytic character of their boundaries.

Once again assume at first that $\Gamma$ is analytic, and that $D$ is deformed into $D^{*}$ by the variation (29). Let the function $\phi(w)$, given in (21), map $D$ onto $C$, and let

$$
\phi^{*}(w)=b_{-1}^{*} w+b_{0}^{*}+\frac{b_{1}^{*}}{w}+\frac{b_{2}^{*}}{w^{2}}+\cdots, \quad b_{-1}>0,
$$

map $D^{*}$ onto $C$. Then Taylor's formula tells us that

$$
\log \left|\phi^{*}\left(y^{*}\right)\right|=\log \left|\phi^{*}(y)\right|+\operatorname{Re}\left(a \rho^{2} \frac{\phi^{\prime}(y)}{\phi(y)} \frac{1}{y-w_{0}}\right)+o\left(\rho^{2}\right),
$$

where $\phi^{*}(y) / \phi^{*}(y)$ is replaced by $\phi^{\prime}(y) / \phi(y)$ since their difference tends to zero with $\rho$. The left-hand side of $(40)$ vanishes when $y^{*}$ is on $\Gamma^{*}$ (or when $y$ is on $\Gamma$ ), so the harmonic function which is the real part of

$$
H\left(y, w_{0}\right)=\log \phi^{*}(y)+a \rho^{2} \frac{\phi^{\prime}(y)}{\phi(y)} \frac{1}{y-w_{0}}
$$

has values of order $o\left(\rho^{2}\right)$ when $y$ is on $\Gamma . H\left(y, w_{0}\right)$ has a logarithmic singularity at $y=\infty$ and a simple pole at $y=w_{0}$. The function 


$$
K\left(y, w_{0}\right)=a \rho^{2} \frac{\phi^{\prime}\left(w_{0}\right)^{2}}{\phi\left(w_{0}\right)} \frac{1}{\phi(y)-\phi\left(w_{0}\right)}-\bar{a} \rho^{2} \frac{\phi^{\prime}\left(w_{0}\right)^{2}}{\phi\left(w_{0}\right)} \frac{\phi(y)}{1-\phi(y) \phi\left(w_{0}\right)}
$$

has only a simple pole at $y=w_{0}$ and has there the same residue as $H\left(y, w_{0}\right)$. $\operatorname{Re} K\left(y, w_{0}\right)$ has value zero when $y$ is on $\Gamma$, so the function $\operatorname{Re}(H-K)$ has only the logarithmic singularity at infinity and boundary values of order $o\left(\rho^{2}\right)$. Thus $\operatorname{Re}(H-K)$ differs from the Green's function of $D, \log |\phi(y)|$, by terms of order $o\left(\rho^{2}\right)$, that is,

$$
\begin{aligned}
\log |\phi(y)|= & \log \left|\phi^{*}(y)\right| \\
& +\operatorname{Re}\left\{a \rho^{2}\left(\frac{\phi^{\prime}(y)}{\phi(y)} \frac{1}{y-w_{0}}-\frac{\phi^{\prime}\left(w_{0}\right)^{2}}{\phi\left(w_{0}\right)} \frac{1}{\phi(y)-\phi\left(w_{0}\right)}\right)\right. \\
& \left.+\bar{a} \rho^{2} \frac{\phi^{\prime}\left(w_{0}\right)^{2}}{\Phi\left(w_{0}\right)} \frac{\phi(y)}{1-\phi(y) \phi\left(w_{0}\right)}\right\}+o\left(\rho^{2}\right) .
\end{aligned}
$$

We may complete this harmonic function to an analytic function to obtain

$$
\begin{aligned}
\log \frac{\phi^{*}(y)}{\phi(y)}= & -a \rho^{2}\left(\frac{\phi^{\prime}(y)}{\phi(y)} \frac{1}{y-w_{0}}-\frac{\phi^{\prime}\left(w_{0}\right)^{2}}{\phi\left(w_{0}\right)} \frac{1}{\phi(y)-\phi\left(w_{0}\right)}\right) \\
& -\bar{a} \rho^{2} \frac{\phi^{\prime}\left(w_{0}\right)^{2}}{\Phi\left(w_{0}\right)} \frac{\phi(y)}{1-\phi(y) \phi\left(w_{0}\right)}+i \rho^{2} C\left(w_{0}\right)+o\left(\rho^{2}\right)
\end{aligned}
$$

where $C\left(w_{0}\right)$ is a real function of $w_{0}$ alone. To determine $C\left(w_{0}\right)$, let $y$ tend to infinity. Then since $\log b_{-1}^{*}$ is real, we get

$$
C\left(w_{0}\right)=-\operatorname{Im} \bar{a} \frac{\bar{\phi}^{\prime}\left(w_{0}\right)^{2}}{\bar{\phi}\left(w_{0}\right)^{2}}=\frac{1}{2 i}\left\{a \frac{\phi^{\prime}\left(w_{0}\right)^{2}}{\phi\left(w_{0}\right)^{2}}-\bar{a} \frac{\bar{\phi}^{\prime}\left(w_{0}\right)^{2}}{\bar{\phi}\left(w_{0}\right)^{2}}\right\} .
$$

Applying the exponential function to both sides of (44) gives us $\delta \phi(y)$, and then, using (23), we obtain the variation formula (32).

In this second proof, we assumed $\Gamma$ to be analytic. If $\Gamma$ is not analytic, we can approximate $\Gamma$ uniformly by a sequence of analytic curves $\Gamma_{n}$ for which (32) holds as a first variation. The remainder terms of order $o\left(\rho^{2}\right)$, which were neglected, are all composed only of the mapping functions and their derivatives which converge uniformly to the mapping functions for $D$ and its derivatives, and hence remain of order $o\left(\rho^{2}\right)$. We may then use formulas (33) to (37) for arbitrary domains.

Another class of functions which we shall consider in this study is the class of functions

$$
f_{0}(z)=z+c+a_{1} / z+a_{2} / z^{2}+\cdots,
$$

analytic and schlicht in $C$, and mapping $C$ onto a domain not containing the origin. The variation formula for $\delta f_{0}$ can be obtained in a manner completely analogous to that described above. In this case, we use the special 
variation which leaves the origin fixed:

$$
w^{*}=w+a \rho^{2} w / w_{0}\left(w-w_{0}\right) .
$$

In this way, we find

$$
\begin{aligned}
\delta f_{0}(x)= & \frac{a \rho^{2}}{2}\left[\frac{2}{f_{0}(x)-f_{0}\left(z_{0}\right)}+\frac{2}{f_{0}\left(z_{0}\right)}\right. \\
& \left.+\left\{\frac{z_{0}+x}{z_{0}-x} x f_{0}^{\prime}(x)+f_{0}(x)\right\} \frac{1}{z_{0}^{2} f_{0}\left(z_{0}\right)^{2}}\right] \\
& +\frac{\bar{a} \rho^{2}}{2}\left[\frac{1+x \bar{z}_{0}}{1-x \bar{z}_{0}} x f_{0}^{\prime}(x)+f_{0}(x)\right] \frac{1}{\bar{z}_{0}^{2} \bar{f}_{0}^{\prime}\left(z_{0}\right)^{2}}
\end{aligned}
$$

for any $z_{0}$ in $C$.

3. We now pose the problem of finding the function of the class for $S$ which $\left|b_{n}\right|$ is a maximum. This problem has meaning since $S$ is a normal family.

If $\phi(w)$ is in $S$, the $e^{i \alpha} \phi\left(e^{-i \alpha} w\right), \alpha$ real, is also in $S$, and has as its $n$th coefficient $e^{i(n+1) \alpha} b_{n}$. If $\left|b_{n}\right|$ is a maximum, we can choose $\alpha$ so that $e^{i(n+1) \alpha} b_{n}$ is real; thus we can rephrase our problem to seek the maximum of $\operatorname{Re} b_{n}$, where the extremal $b_{n}$ will be real.

From (36), we obtain for each point $z$ in $C$,

$$
\begin{gathered}
\operatorname{Re} \delta b_{n}=\operatorname{Re} a \rho^{2}\left\{\left[\frac{1}{n} z^{2} P_{n}^{\prime}(z)+(n-1) b_{n-1} z+(n+1) b_{n}+(n-1) b_{n-1} \frac{1}{z}\right.\right. \\
\left.\left.+\frac{1}{n} \frac{1}{z^{2}} \bar{P}_{n}^{\prime}\left(\frac{1}{z}\right)\right] \frac{1}{z^{2} f^{\prime}(z)^{2}}+\sum_{\nu=-1}^{n-2} \nu b_{\nu} f(z)^{n-\nu-2}\right\}
\end{gathered}
$$

where $a$ is an arbitrary constant with $|a|=1$ and $w=f(z)$ is the inverse of $z=\phi(w)$. If $b_{n}$ is an extremal, then $\operatorname{Re} \delta b_{n} \leqq 0$; the arbitrariness of $a$ now allows us to conclude that for all $z$ in $C$,

$$
\begin{array}{r}
\frac{1}{n} z^{2} P_{n}^{\prime}(z)+(n-1) b_{n-1} z+(n+1) b_{n}+(n-1) \bar{b}_{n-1} \frac{1}{z}+\frac{1}{n} \frac{1}{z^{2}} \bar{P}_{n}^{\prime}\left(\frac{1}{z}\right) \\
=-z^{2} f^{\prime}(z)^{2} \sum_{\nu=-1}^{n-2} \nu b_{\nu} f(z)^{n-\nu-2} .
\end{array}
$$

For convenience we shall set

$$
\begin{aligned}
Q_{n}(z)= & \frac{1}{n} z^{2} P_{n}^{\prime}(z)+(n-1) b_{n-1} z+(n+1) b_{n} \\
& +(n-1) b_{n-1} \frac{1}{z}+\frac{1}{n} \bar{P}_{n}^{\prime}\left(\frac{1}{z}\right)
\end{aligned}
$$


and

$$
S_{n}(w)=-\sum_{\nu=-1}^{n-2} \nu b_{\nu} w^{n-2-\nu} .
$$

Since $Q_{n}(z)$ is analytic in the whole plane and agrees with $z^{2} f^{\prime}(z)^{2} S_{n}(f(z))$ in $C, Q_{n}(t)$ can be used to extend $z^{2} f^{\prime}(z)^{2} S_{n}(f(z))$ to the whole plane. Thus (50) is valid for all $z$, and by separting variables, taking the square root, and integrating both sides of $(50)$, we find

$$
\int\left(Q_{n}(z)\right)^{1 / 2} \frac{d z}{z}=\int\left(S_{n}(w)\right)^{1 / 2} d w .
$$

These integrals are analytic functions except at the zeros and poles of the rational function $Q_{n}(z)$ and the zeros of the polynomial $S_{n}(w)$, which are actually only a finite number of points. Thus $w$ is determined as an analytic function of $z$ with branch points at most at the zeros and poles of $Q_{n}(z)$ and $S_{n}(w)$. We conclude that the circle $\gamma$ is mapped onto a finite number of analytic arcs by the extremal function $w=f(z)$ and that these arcs join at most at the points corresponding to the zeros or poles of $Q_{n}(z)$ or $S_{n}(w)$. If several analytic arcs come together at a point, their tangents will lie along rays that meet at equal angles at that point, the number of such rays depending upon the order of the branch point of (52).

The information that the extremal domains are bounded by analytic arcs enables us to use the Julia formula directly, since the Poisson integral can be applied to these cases and the considerations of $\$ 2$ are all valid. Consequently (28) may now be applied to give

$$
\operatorname{Re} \delta b_{n}=\oint_{\gamma} Q_{n}(z) \frac{1}{2 \pi i} \frac{\delta w d w}{z^{2} f^{\prime}(z)^{2}} .
$$

According to (50), however, we see that

$$
\frac{Q_{n}(z)}{z^{2} f^{\prime}(z)^{2}}=S_{n}(w)
$$

which tells us that the integrand in (53) is a function of $w$ alone; namely,

$$
\operatorname{Re} \delta b_{n}=\frac{1}{2 \pi i} \oint_{\Gamma} S_{n}(w) \delta w d w .
$$

We shall now deduce another important property of the extremal domain $\widetilde{D}$; it has no exterior points. This means that the boundary is made up of a slit composed of a finite number of analytic arcs. To prove this assertion, let us assume that the domain does have exterior points. Then there is a boundary arc with interior points on one side and exterior points on the other side. If 
we vary this boundary arc slightly toward the interior of $\tilde{D}$, we define a certain $\delta w$. We can, however, make this variation small enough so that the variation $-\delta w$ will move the arc into the exterior of $\tilde{D}$ without crossing any other parts of $\Gamma$. Since $S_{n}(w)$ is not identically zero, we can localize the arbitrary (but small) shift and be sure that $\operatorname{Re} \delta b_{n}$ is not zero. But whether it is positive or negative, the shift $-\delta w$ will give $\operatorname{Re} \delta b_{n}$ the opposite sign, meaning that $b_{n}$ could not be an extremal. This contradiction proves that $\tilde{D}$ is a slit domain.

$Q_{n}(z)$ assumes only non-negative values on $\gamma$. For, using (12), we may write (53) in the form

$$
\operatorname{Re} \delta b_{n}=-\frac{1}{2 \pi} \oint_{\gamma} Q_{n}(z) \delta n \frac{d \phi}{d s} d \phi
$$

where $(d \phi / d s) d \phi$ is always positive. Suppose that $Q_{n}\left(z_{0}\right)$ is negative for $z_{0}$ on $\gamma$. Then there is a small neighborhood $\gamma^{\prime}$ of $z_{0}$ on which $Q_{n}(z)$ is negative. We can separate the two edges of the boundary slit $\Gamma$, one of which corresponds to $\gamma^{\prime}$, in such a way that one edge stays fixed and the other edge, actually corresponding to $\gamma^{\prime}$, is shifted into the interior of $D$. This variation defines a positive $\delta n$ since the entire shift is into the interior of $D$. Since $Q_{n}(z)$ is negative on the $\operatorname{arc} \gamma^{\prime},(56)$ says that $\operatorname{Re} \delta b_{n}$ is positive, which shows that $b_{n}$ did not have maximum real part. Thus $Q_{n}(z)$ is always non-negative on $\gamma$.

The extremal domain $D$ is thus the whole plane with the exception of a slit $\Gamma$ composed of a finite number of analytic arcs, the ends of which either join with other such arcs as forks or terminate as tips, not meeting any other boundary arcs. At the point $z_{0}$ of $\gamma$ which maps into such a tip, we see that the angle $\pi$ made by the arcs of $\gamma$ meeting at $z_{0}$ maps into an angle of $2 \pi$ at the tip. Thus $f^{\prime}(z)$ must be zero at each point of $\gamma$ mapping into a tip of $\Gamma$. From (50), we see that at each such tip, $Q_{n}(z)$ must have a double zero. If both sides of (50) are multiplied by $z^{n+1}$, the left side becomes a polynomial of degree $2 n+2$, and hence can have at most $n+1$ double zeros. We have thus proved that $\Gamma$ can have at most $n+1$ free tips.

Since $Q_{n}(z)$ is real and non-negative on $\gamma$, we can divide $\gamma$ into a finite number of parts, $\gamma_{i}$, such that $Q_{n}(z)$ is definitely positive for $z$ on $\gamma_{i}$. Equation (54) can be rewritten as

$$
S_{n}(w) d w^{2}=-Q_{n}\left(e^{i \phi}\right)(d \phi)^{2} .
$$

We now define a new parameter on $\gamma_{i}$ (or its image $\Gamma_{i}$ ) by setting

$$
d t^{2}=Q_{n}\left(e^{i \phi}\right)(d \phi)^{2} .
$$

This gives us a differential equation that must be satisfied by the extremal $\operatorname{arcs} \Gamma_{i}$; namely

$$
S_{n}(w(t))(d w / d t)^{2}+1=0
$$


$t$ being a real parameter on $\Gamma_{i}$. This type of equation was first obtained by Schiffer [12] using a special boundary variation.

Let us now consider the special case when $n$ is equal to one; then

$$
Q_{1}(z)=z^{2}+2 b_{1}+1 / z^{2}, \quad S_{1}(w)=1,
$$

and (50) specializes to

$$
z^{2}+2 b_{1}+1 / z^{2}=z^{2} f^{\prime}(z)^{2} .
$$

Since $\Gamma$ must have at least one free tip, we know that $f^{\prime}(z)$ has at least one zero on $\gamma$, say at $e^{i \alpha}$. Then substituting $e^{i \alpha}$ into the left side of (61) yields

$$
b_{1}=-\frac{1}{2}\left(e^{2 i \alpha}+e^{-2 i \alpha}\right)=-\cos 2 \alpha .
$$

Consequently $\left|b_{1}\right| \leqq 1$, and putting $b_{1}=1$ into (61) leaves a perfect square on the left allowing us to integrate and get

$$
f(z)=z-1 / z
$$

a mapping of $\gamma$ onto a linear slit of length 4 and center at the origin (Faber [3], Bieberbach [1]). Thus $\left|b_{1}\right| \leqq 1$ with equality only in the case when $z=\phi(w)$ is the inverse of the function

$$
f(z)=z-\frac{\epsilon}{z}, \quad|\epsilon|=1 .
$$

We next consider the problem of maximizing the modulus of the third coefficient $b_{3}$ for the functions in the class $S$. To do so, we shall first maximize the expression $\left|b_{1}^{2}+2 b_{3}\right|$. If we set $\phi^{*}(w)=e^{i \beta} \phi\left(e^{-i \beta} w\right)$, we get $b_{1}^{*}+2 b_{3}^{*}$ $=e^{4 i \beta}\left(b_{1}^{2}+2 b_{3}\right)$; thus $\beta$ may always be chosen such that the extremal $b_{1}^{2}+2 b_{3}$ is real, and we can consider the equivalent problem of maximizing $\operatorname{Re}\left(b_{1}^{2}+2 b_{3}\right)$. From (36), we obtain

$$
\begin{aligned}
\operatorname{Re} \delta\left(b_{1}^{2}+2 b_{3}\right)= & 2 \operatorname{Re}\left(b_{1} \delta b_{1}+\delta b_{3}\right) \\
= & 2 \operatorname{Re} a \rho^{2}\left\{\left[\frac{1}{3} z^{2} P_{3}^{\prime}(z)+z^{2} P_{1}^{\prime}(z) b_{1}+2 b_{2} z+2\left(b_{1}^{2}+2 b_{3}\right)\right.\right. \\
& +2 \bar{b}_{2} \frac{1}{z}+\frac{1}{z^{2}} \bar{P}_{1}^{\prime}\left(\frac{1}{z}\right) \bar{b}_{1} \\
& \left.\left.+\frac{1}{3} \frac{1}{z^{2}} \bar{P}_{3}^{\prime}\left(\frac{1}{z}\right)\right] \frac{1}{z^{2} f^{\prime}(z)^{2}}-f(z)^{2}\right\} \\
= & 2 \operatorname{Re} a \rho^{2}\left[Q(z) \frac{1}{z^{2} f^{\prime}(z)^{2}}-f(z)^{2}\right],
\end{aligned}
$$


where $z$ is any point of $C$ and $a$ is any constant with $|a|=1, Q(z)$ being defined as that rational function in (65) multiplying $1 / z^{2} f^{\prime}(z)^{2}$. In order for $b_{1}^{2}+2 b_{3}$ to be an extremal, (65) implies that

$$
Q(z)=z^{2} f^{\prime}(z)^{2} f(z)^{2} .
$$

$Q(z)$ is real on $\gamma$ and, as before, $Q(z)$ must be non-negative on $\gamma$ and must lead to slit domains as extremals. Corresponding to (59), the extremal curve consists of a finite number of analytic arcs $\Gamma_{i}$, each of which satisfies an equation of the form

$$
\left(\frac{d w}{d t}\right)^{2} w^{2}+1=0
$$

for a suitably chosen real parameter $t$ on $\Gamma_{i}$.

The differential equation (67) can be integrated directly to give $w^{2}$ $=2 i t+c$, where $c$ is the constant of integration. Thus for $z$ on $\gamma, f(z)^{2}-c$ is purely imaginary, and may be continued to $|z| \leqq 1$ by the Schwarz reflection principle. This leads to the analytic function $g(z)$ with

$$
\begin{array}{ll}
g(z)=f(z)^{2}-c & \text { for }|z| \geqq 1, \\
g(z)=\frac{\text { for }|z|<1 .}{-f(1 / z)^{2}-c} & \text { fo }
\end{array}
$$

If we introduce the expansion (1) for $f(z)$ about infinity, we obtain

$$
g(z)=z^{2}+2 a_{1}-c+\frac{2 a_{2}}{z}+\frac{a_{1}^{2}+2 a_{3}}{z^{2}}+\cdots .
$$

Since $g(z)$ has the singular part $z^{2}$ at infinity, (68) says that $g(z)$ has the singular part $-1 / z^{2}$ at the origin and is otherwise regular. Thus $g(z)$ is a rational function which has the form

$$
g(z)=z^{2}+\left(2 a_{1}-c\right)-\frac{1}{z^{2}} .
$$

Comparison of the coefficients in (69) and (70) shows that $a_{2}=0$ and $a_{1}^{2}+2 a_{3}$ $=-1$ for the extremal function. Recalling that $z=\phi(w)$ is the inverse of $w=f(z)$, we find that $a_{1}=-b_{1}, a_{3}=-b_{3}-b_{1}^{2}$. Therefore for the extremal function, $b_{1}^{2}+2 b_{3}=1$. This means that as $\phi(w)$ varies through the class $S$,

$$
\left|b_{1}^{2}+2 b_{3}\right| \leqq 1 \text { or }\left|b_{3}\right| \leqq \frac{1}{2}\left(1+\left|b_{1}\right|^{2}\right) .
$$

Since we have already shown that $\left|b_{1}\right| \leqq 1$ with the extremal function (64), this implies that $\left|b_{3}\right| \leqq 1$ with the same extremal function (64).

Since $\left|b_{2}\right|$ is maximum in the inverse of the function (4), precise bounds are now known for the quantities $\left|b_{1}\right|,\left|b_{2}\right|$, and $\left|b_{3}\right|$. 
We shall now study a class of extremal functions of the differential equation (50). Let us denote by $E_{q}$ the domain consisting of the whole $w$-plane with the exception of $q(q \geqq 2)$ symmetric radial slits each of length (2) $2 / q$ emanating from the origin (one ray will be assumed to lie along the positive real axis). The function which maps $E_{q}$ conformally upon the exterior of the unit circle will be denoted by

$$
\phi_{q}(w)=w+\frac{c_{1}^{(q)}}{w}+\frac{c_{2}^{(q)}}{w^{2}}+\cdots .
$$

The inverse of $\phi_{q}(w)$ is given explicitly by

$$
w=f_{q}(w)=z\left(1+z^{-q}\right)^{2 / q} .
$$

It will now be proved that if $q$ is a divisor of $n+1$, that is, if $q k=n+1$ for some integer $k$, then $f_{q}(z)$ satisfies (50) for this $n$.

Let us first evaluate $c_{\nu}^{(q)}$. If $\Gamma^{\prime}$ is a large circle $|w|=R$, and $\gamma^{\prime}$ the image of $\Gamma^{\prime}$ under $\phi_{q}(w)$, then

$$
\begin{aligned}
-\nu c_{\nu}^{(q)} & =\frac{1}{2 \pi i} \oint_{\Gamma^{\prime}} w^{\nu} \phi_{q}^{\prime}(w) d w=\frac{1}{2 \pi i} \oint_{\gamma^{\prime}} f_{q}(z)^{\nu} d z \\
& =\frac{1}{2 \pi i} \oint_{\gamma^{\prime}} z^{\nu}\left(1+z^{-q}\right)^{2 \nu / q} d z .
\end{aligned}
$$

Expanding $\left(1+z^{-q}\right)^{2 v / q}$ by the binomial theorem and using the residue theorem, we obtain

$$
c_{\nu}^{(q)}=-(1 / \nu) C_{2 \nu / q, k} \quad \text { if } q k=\nu+1, k=\text { integer, }
$$

and $c_{\nu}^{(q)}=0$ when $q$ does not divide $\nu+1$. In particular, if $q$ divides $n+1$, then $q$ does not divide $n$, and we have $c_{n-1}^{(a)}=0$; then we may neglect the terms in $z$ and $1 / z$ on the left side of $(50)$.

In general, the Faber polynomial of degree $n$ for the function $z=\phi(w)$ is the polynomial part of the $n$th power of the inverse function $w=f(z)$ (Schur $[15])$. We shall introduce the notation $[g(z)]_{z}$ to represent the polynomial part of $g(z)$ when it is expanded in powers of $z$ in the neighborhood of infinity. Hence, we have

$$
P_{n}(z)=\left[f_{q}(z)^{n}\right]_{z}=\sum_{v=0}^{k-1} C_{2 n / q, \nu z^{n-\nu q},}, \quad k q=n+1,
$$

and

$$
z^{2} P_{n}^{\prime}(z)=\sum_{\nu=0}^{k-1}(n-\nu q) C_{2 n / q, \nu z^{q(k-\nu)}}=z^{2}\left[\frac{d}{d z}\left(f_{q}(z)^{n}\right)\right]_{z} .
$$

A fact that will be useful later is 


$$
z^{2} P_{n}^{\prime}(z)=\left[z^{2} \frac{d}{d z}\left(f_{q}(z)^{n}\right)\right]_{z}-n c_{n}^{(q)} .
$$

According to (76), the expression $z^{2} P_{n}^{\prime}(z)+\left(1 / z^{2}\right) \bar{P}_{n}^{\prime}(1 / z)$ involves only integral powers of $z^{q}$ and the terms can be paired so that each is of the form $z^{q i}+z^{-q j}$. Since solving (73) for $z^{q}$ gives

$$
\begin{aligned}
z^{q} & =\frac{1}{2}\left[\left(w^{q}-2\right)+\left(w^{2 q}-4 w^{q}\right)^{1 / 2}\right], \\
z^{-q} & =\frac{1}{2}\left[\left(w^{q}-2\right)-\left(w^{2 q}-4 w^{q}\right)^{1 / 2}\right],
\end{aligned}
$$

the terms $z^{q j}+z^{-q j}$ are polynomials in $w$. Thus $\phi_{q}(w)^{2} P_{n}^{\prime}\left(\phi_{q}(w)\right)$ $+\phi_{q}(w)^{-2} \bar{P}_{n}^{\prime}\left(\phi_{q}(w)^{-1}\right)$ is a polynomial in $w$. However, $\phi_{q}(w)^{-2} \bar{P}\left(\phi_{q}(w)^{-1}\right)$ will have only negative powers of $w$, allowing us to say

$$
\phi_{q}(w)^{2} P_{n}^{\prime}\left(\phi_{q}(w)\right)+\phi_{q}(w)^{-2} \bar{P}_{n}^{\prime}\left(\phi_{q}(w)^{-1}\right)=\left[\phi_{q}(w)^{2} P_{n}^{\prime}\left(\phi_{q}(w)\right)\right]_{w} .
$$

From (77), we obtain

$$
\frac{1}{n} z^{2} P_{n}^{\prime}(z)=\left[z\left(z^{q / 2}+z^{-q / 2}\right)^{(2 n / q)-1}\left(z^{q / 2}-z^{-q / 2}\right)\right]_{z}-c_{n}^{(q)}
$$

$$
=z\left(z^{q / 2}+z^{-q / 2}\right)^{(2 n / q)-1}\left(z^{q / 2}-z^{-q / 2}\right)-c_{n}^{(q)}-\sum_{\nu=1}^{\infty} A_{\nu} z^{-\nu}
$$

where the $A_{\nu}$ are chosen so as to leave only polynomial terms. If we substitute $\phi_{q}(w)$ for $z$ in (80), the last sum will contribute only negative powers of $w$; thus

$$
\frac{1}{n}\left[\phi_{q}(w) P_{n}^{\prime}\left(\phi_{q}(w)\right)\right]_{w}=\left[\phi_{q}(w) w^{n}\left(1-4 w^{-q}\right)^{1 / 2}\right]_{w}-c_{n}^{(q)} .
$$

Since

$$
\phi_{q}(w) / \phi_{q}^{\prime}(w)=w\left(1-4 w^{-q}\right)^{1 / 2}
$$

we get

$$
\begin{aligned}
\frac{1}{n}\left[\phi_{q}(w) P_{n}^{\prime}\left(\phi_{q}(w)\right)\right]_{w} \\
\quad=\left[\frac{\phi_{q}(w)^{2}}{\phi_{q}^{\prime}(w)^{2}} w^{n-1} \phi_{q}^{\prime}(w)\right]_{w}-c_{n}^{(q)} \\
=\frac{-\phi_{q}(w)^{2}}{\phi_{q}^{\prime}(w)^{2}}\left\{\sum_{\nu=0}^{k-1}(q \nu-1) c_{q \nu-1}^{(q)} w^{q(k-\nu)-2}\right\}-(n+1) c_{n}^{(q)} .
\end{aligned}
$$


In view of (79), this equation is the same as (50) when expressed in terms of $z$, proving that $\phi_{q}(w)$ is a solution of (50) if $q$ divides $n+1$.

Let us denote by $S\left[b_{1}, b_{2}, \cdots, b_{n-1}\right]$ the subclass of $S$ consisting only of those univalent functions having the first $n-1$ coefficients in the expansion (2) about infinity fixed at those values indicated in the brackets. We shall also let $T\left[a_{1}, a_{2}, \cdots, a_{n-1}\right]$ represent the subclass of $T$ having the first $n-1$ coefficients fixed at the values indicated. The next problem to be considered is the determination of the extreme value of $\operatorname{Re}\left(b_{n}\right)$ as $\phi$ varies through the class $S\left[c_{1}^{(q)}, c_{2}^{(q)}, \cdots, c_{n-1}^{(q)}\right], n=q k-1$ for some positive integer $k$, rather than for all $\phi$ in $S$.

The coefficients of $b_{n}$ of $\phi$ and $a_{n}$ of $f$, the inverse of $\phi$, are related by the polynomial relation

$$
a_{n}=-b_{n}+M\left(b_{1}, b_{2}, \cdots, b_{n-1}\right),
$$

where $M$ is a polynomial in the $n-1$ variables $b_{1}, \cdots, b_{n-1}$. Since we have fixed the first $n-1$ coefficients as $b_{i}=c_{i}^{(q)}, i=1, \cdots, n-1, M\left(b_{1}, \cdots, b_{n-1}\right)$ is a fixed constant and $\operatorname{Re} a_{n}$ and $\operatorname{Re} b_{n}$ achieve their extreme values simultaneously. Also $a_{1}, a_{2}, \cdots, a_{n-1}$ are determined completely by $b_{1}, b_{2}, \cdots$, $b_{n-1}$, so the first $n-1$ coefficients of $f$ are also fixed.

Using the method of strips, Teichmueller [16] proved the following theorem: Among all functions $f$ of $T\left[a_{1}, \cdots, a_{n-1}\right]$, the function $w=f(z)$ which maps the unit circle onto a slit composed of arcs satisfying the differential inequality $P(w) d w^{2}>0, P(w)=\alpha w^{n-1}+\cdots$ being a polynomial of degree $n-1$, gives the maximum value to $\operatorname{Re} \alpha a_{n}$, and this function is unique.

We have shown that $f_{q}(z)$ satisfies $(50)$, so for $z=e^{i \theta}$, we may set

$$
P(w)(d w)^{2}=S_{n}(w)(d w)^{2}=-Q_{n}\left(e^{i \theta}\right)(d \theta)^{2} .
$$

The function $Q_{n}\left(e^{i \theta}\right)$ may be evaluated using (76) and (74), giving us

$$
Q_{n}\left(e^{i \theta}\right)=\frac{2}{n} \sum_{\nu=0}^{k-1}(n-\nu q) C_{2 n / q, \nu} \cos [q(k-\nu) \theta]-\frac{n+1}{n} C_{2 n / q, k}
$$

As a function of $\theta, Q_{n}\left(e^{i \theta}\right)$ clearly assumes its maximum value for $\theta=0$. Remembering that $q k=n+1$, we may evaluate this maximum value:

$$
n Q_{n}(1)=2 \sum_{\nu=0}^{k-1}[q(k-\nu)-1] C_{2 k-2 / q, \nu}-q k C_{2 k \rightarrow 2 / q, k} .
$$

We shall now show that $n Q_{n}(1)=0$. Let $A_{\nu+1}=(\nu+1) q C_{2 k-2 / q, \nu+1}$. Then from the definition of the binomial coefficients, we obtain

$$
A_{\nu+1}=[q(k-\nu)-1] C_{2 k-2 / q, \nu}+(q k-1) C_{2 k-2 / q, \nu}
$$

Hence

$$
A_{\nu+1}-A_{\nu}=2[q(k-\nu)-1] C_{2 k-2 / q, \nu}
$$


Summing these differences on $\nu$ from 0 to $k-1$, and noticing that $A_{0}=0$, we get

$$
2 \sum_{\nu=0}^{k-1}[q(k-\nu)-1] C_{2 k-2 / q, \nu}=q k C_{2 k-2 / q, k}
$$

for all integers $q$ and $k$, proving that $n Q_{n}(1)=0$. Then $S_{n}(w) d w=-Q_{n}\left(e^{i \theta}\right) d \theta^{2}$ $\geqq 0$ for $w$ on the boundary of $E_{q}$ and as before, we have equality for only a finite number of points. Since $S_{n}(w)=w^{n-1}+\cdots$, we can set $\alpha=+1$ in Teichmueller's theorem, and conclude that the $n$th coefficient in the expansion of $f_{q}(z)$ about infinity, call it $a_{n}^{(q)}$, satisfies the inequality

$$
\operatorname{Re}\left(a_{n}\right)<\operatorname{Re}\left(a_{n}^{(q)}\right),
$$

where $a_{n}$ is the $n$th coefficient in the expansion of any other function of the class $T\left[a_{1}^{(q)}, a_{2}^{(q)}, \cdots, a_{n-1}^{(q)}\right]$. The relation $(q<1)$ now assures us that $\operatorname{Re} c_{n}^{(q)}$ will be an absolute minimum among all $\operatorname{Re} b_{n}$, where $b_{n}$ is the $n$th coefficient in the expansion of any function in $S\left[c_{1}^{(q)}, c_{2}^{(q)}, \cdots, c_{n-1}^{(q)}\right]$.

We have seen that $-c_{n}^{(q)}=(1 / n) C_{2 n / q, k}, n=q k-1$. Let us keep $n$ fixed and find that $q$ for which $-c_{n}^{(q)}$ is a maximum. Setting $\lambda=2 n /(n+1)$, we see that $-c_{n}^{(q)}=(1 / n) C_{k \lambda, k}$. We shall show that $C(k, \lambda)=C_{k \lambda, k}$ is an increasing function of $k$ which means that $-c_{n}^{(q)}$ is a decreasing function of $q$ and will assume its largest value for the smallest possible value of $q$, hence the least prime divisor of $n+1$.

In general, we have

$$
\frac{C(k+1, \lambda)}{C(k, \lambda)}=\lambda \prod_{\nu=1}^{k} r_{\nu}, \quad r_{\nu}=\frac{(k+1) \lambda-\nu}{k \lambda+1-\nu} .
$$

Since $\lambda \geqq 1$, each $r_{\nu} \geqq 1$ for $\nu=1,2, \cdots, k$; thus $C(k+1, \lambda) \geqq C(k, \lambda)$.

We have shown that $-c_{n}^{(q)}$ is largest when $q$ is the least prime divisor of $n+1$. It has been proved that as $\phi$ varies through the class $S,\left|b_{1}\right| \leqq-c_{1}^{(2)}$, $\left|b_{2}\right| \leqq-c_{2}^{(3)}$, and $\left|b_{3}\right| \leqq-c_{3}^{(2)}$, equality holding only when $\phi(w)=e^{i \alpha} \phi_{q}\left(e^{-i \alpha} w\right)$, $q=2,3$, and 2 , respectively. This leads us to the general conjecture that for functions in the class $S,\left|b_{n}\right| \leqq-c_{n}^{(q)}$ where $q$ is the least prime divisor of $n+1$, equality holding only when $\phi(w)=e^{i \alpha} \phi_{q}\left(e^{-i \alpha} w\right)$.

Rough estimates for $\left|b_{n}\right|$ can be obtained using the Cauchy integral formula. We have

$$
-n b_{n}=\frac{1}{2 \pi i} \oint_{\Gamma^{\prime}} w^{n} \phi^{\prime}(w) d w=\frac{1}{2 \pi i} \oint_{\gamma^{\prime}} f(z)^{n} d z,
$$

$\Gamma^{\prime}$ and $\gamma^{\prime}$ being contours in $D$ and $C$ respectively which are close to $\Gamma$ and $\gamma$. Under the normalized mapping $f(z)$, the unit circle goes into a domain which lies entirely within a circle of radius 2 about the origin (Bieberbach [1]). Thus we get the estimate 


$$
\left|b_{n}\right| \leqq 2^{n} / n \text {. }
$$

Let us compare this estimate with the above conjecture in the case of odd coefficients $b_{2 n-1}$. The conjecture states that

$$
\left|b_{2 n-1}\right| \leqq-c_{2 n-1}^{(2)}=\frac{1}{2 n-1} C_{2 n-1, n}=\frac{(2 n-2) !}{n !(n-1) !} .
$$

Applying Stirling's formula to the factorials in (95) yields asymptotically

$$
-c_{2 n-1}^{(2)} \sim \frac{e}{\pi^{1 / 2}} \frac{2^{2 n-2}}{n\left(n^{1 / 2}\right)} ;
$$

this is to be compared with (94), which for odd coefficients becomes $\left|b_{2 n-1}\right|$ $\leqq 2^{2 n-2} /(n-1 / 2)$. We note that this rough estimate differs asymptotically from the conjecture by a factor of $n^{1 / 2}$.

Let us now obtain some further information about the extremal functions in $S$ which have maximal $\left|b_{n}\right|$ using the differential equation (50). Since (50) holds for all values of $z$, the coefficients of $z^{k}$ must agree on both sides. The coefficients of the positive powers of $z$ turn out to be formally the same on both sides, whereas those belonging to negative powers of $z$ yield an infinite number of relations that must be satisfied by the coefficients of an extremal function. As an example, we shall compare the coefficients of $z^{-1}$ on both sides of (50), which amounts to computing the residues on both sides.

We observe first that by differentiating the relation $z=\phi(f(z))$, we obtain

$$
1=-f^{\prime}(z) \sum_{\nu=-1}^{\infty} \nu b_{v} f(z)^{-\nu-1} \text {. }
$$

Hence

$$
Q_{n}(z)=z^{2} f(z)^{n-1} f^{\prime}(z)+z^{2} f^{\prime}(z)^{2} \sum_{\nu=1}^{\infty}(n-2+\nu) b_{n-2+\nu} f(z)^{-\nu} .
$$

The residue of the right-hand side of (98) is now given by

$$
\frac{1}{2 \pi i} \oint z^{2} f(z)^{n-1} d z+\sum_{\nu=1}^{\infty}(n-2+\nu) b_{n-2+\nu} \frac{1}{2 \pi i} \oint z^{2} f(z)^{-\nu} f^{\prime}(z) d z
$$

which may be transformed to the $w$-plane giving

$$
\frac{1}{2 \pi i} \oint \phi(w)^{2} w^{n-1} d w+\sum_{\nu=1}^{\infty}(n-2+\nu) b_{n-2+\nu} \frac{1}{2 \pi i} \oint \frac{\phi(w)^{2}}{\phi^{\prime}(w)} \frac{1}{w^{\nu}} d w .
$$

Since

$$
\frac{\phi(w)^{2}}{\phi^{\prime}(w)}=w^{2}+3 b_{1}+\frac{4 b_{2}}{w}+\cdots
$$


only the terms for $\nu=1$ and $\nu=3$ in the sum in (100) contribute to the residue. Thus

$$
(n-1) b_{n-1}=\sum_{\nu=1}^{n+1} b_{\nu} b_{n \rightarrow p}+3(n-1) b_{1} b_{n-1}+(n+1) b_{n+1}
$$

must be satisfied by the coefficients of any extremal function for maximum $\left|b_{n}\right|$.

The formula given in (102) can also be obtained by making a special variation on the class $S$ which is a generalization of a variation that was introduced by Marty [10] in the study of univalent functions in $|z|<1$. Once again let $f$ be a function in the class $T$ which is the inverse of a function $\phi$ in $S$. Then for any $a,|a|<1$, the function

$$
\begin{aligned}
f_{a}^{*}(z) & =\frac{c_{1}}{\frac{1}{f((z+a) /(1+\bar{a} z))}-\frac{1}{f(1 / \bar{a})}}+c_{2}, \\
c_{1} & =\frac{f^{\prime}\left(\bar{a}^{-1}\right)}{f\left(\bar{a}^{-1}\right)} \frac{\left(1-|a|^{2}\right)}{\bar{a}^{2}}, \\
c_{2} & =-\left(\bar{a}^{-1}\right)\left[1+\frac{\left(|a|^{2}-1\right)}{\bar{a}}\left\{\frac{f^{\prime}\left(\bar{a}^{-1}\right)}{f\left(\bar{a}^{-1}\right)}-\frac{f^{\prime \prime}\left(\bar{a}^{-1}\right)}{f^{\prime}\left(\bar{a}^{-1}\right)}\right\}\right]
\end{aligned}
$$

will also be in $T, c_{1}$ and $c_{2}$ having been chosen to give the correct normalization.

There are now two possibilities for proceeding: first, we could expand $f_{a}^{*}(z)$ in powers of $a$; second, we could expand it in powers of $z$. We note that as $a$ tends to zero, $f_{a}^{*}(z)$ converges to $f(z)$, and for small values of $a, f_{a}^{*}(z)$ may be considered as a variation from $f(z)$ in the class $T$. Expanding in powers of $a$, and considering only the first variation (neglecting terms of order $\left.|a|^{2}\right)$ we obtain

$$
\delta f(z)=a\left(f^{\prime}(z)-1\right)+\bar{a}\left[f(z)^{2}-z^{2} f^{\prime}(z)+3 b_{1}\right]+O\left(|a|^{2}\right) .
$$

According to (23), the corresponding variation formula for $\phi(w)$ is given by

$$
-\delta \phi(w)=a\left(1-\phi^{\prime}(w)\right)+\bar{a}\left[w^{2} \phi^{\prime}(w)-\phi(w)^{2}+3 b_{1}\right]+O\left(|a|^{2}\right) .
$$

By comparing the coefficients of $w$ on both sides of (105), we arrive at

$$
\begin{aligned}
\operatorname{Re} \delta b_{n}= & \bar{a}\left(\sum_{\nu=1}^{n+1} b_{\nu} b_{n \rightarrow \nu}+(n+1) b_{n+1}+3(n-1) b_{n-1}-(n-1) b_{n-1}\right) \\
& +O\left(|a|^{2}\right) .
\end{aligned}
$$

If $b_{n}$ is an extremal for $\operatorname{Re} b_{n}{ }^{*},(106)$ must always have the same sign, and 
since the argument of $\bar{a}$ is arbitrary, the coefficient of $\bar{a}$ must vanish, again giving us the relation (102).

On the other hand, if we expand $f_{a}^{*}(z)$ in powers of $z$, we obtain

$$
\begin{aligned}
f_{a}^{*}(z) & =z+\frac{a_{1}^{*}}{z}+\frac{a_{2}^{*}}{z^{2}}+\cdots \\
a_{1}^{*} & =\left[\frac{1}{4} \frac{f^{\prime \prime}\left(\bar{a}^{-1}\right)^{2}}{f^{\prime}\left(\bar{a}^{-1}\right)^{2}}-\frac{1}{6} \frac{f^{\prime \prime \prime}\left(\bar{a}^{-1}\right)}{f^{\prime}\left(\bar{a}^{-1}\right)}\right] \frac{\left(|a|^{2}-1\right)^{2}}{\bar{a}^{4}} .
\end{aligned}
$$

Since $f_{a}^{*}(z)$ belongs to $T,\left|a_{1}^{*}\right| \leqq 1$, and replacing $\bar{a}^{-1}$ by $z$ in this inequality yields the following distortion theorem:

$$
\left|2 \frac{f^{\prime \prime \prime}(z)}{f^{\prime}(z)}-3 \frac{f^{\prime \prime}(z)^{2}}{f^{\prime}(z)^{2}}\right| \leqq \frac{12}{\left(|z|^{2}-1\right)^{2}} .
$$

This distortion theorem is precise in the sense that we have equality for the function $f(z)=z+1 / z$ when $z>1$. Similarly, estimates of the higher coefficients $b_{n}$ lead to inequalities on certain polynomials in the $a_{n}{ }^{*}$, which can be expressed in terms of derivatives of $f(z)$. This generates an infinite number of distortion theorems, one for each coefficient $a_{n}$ or $b_{n}$ for which precise estimates are known.

4. Corresponding to each function $f(z)=z+a_{1} / z+a_{2} / z^{2}+\cdots$ in the class $T$, we have a point $p_{n}=\left(a_{1}, a_{2}, \cdots, a_{n}\right)$ of $2 n$-dimensional real euclidean space. The points $p_{n}$ fill out a domain $V_{n}$ as $f$ varies through $T$; we call $V_{n}$ the $n$th coefficient region of $T$. The boundary of $V_{n}$ will be denoted by $B_{n}$; $B_{n}$ is thus a $(2 n-1)$-dimensional manifold. In this section, certain properties of those functions corresponding to points $p_{n}$ on $B_{n}$ will be described.

The domain $V_{n}$ generated by $\left(a_{1}, a_{2}, \cdots, a_{n}\right)$ will remain unchanged if instead of considering the function $f(z)$ in $T$, we consider the function $f_{0}(z)$ $=f(z)+c$, where $c$ is a constant. In particular, we may choose $c$ such that $f_{0}(z)$ $\neq 0$ for $z$ in $C$; this new class of functions $f_{0}$ will be denoted by $T_{0}$. It is well known that $|c| \leqq 2$ (Bieberbach [1]).

We shall now consider the regular points on $B_{n}$; that is, boundary points on part of the surface $B_{n}$ which may be represented locally by an equation

$$
F\left(a_{1}, \bar{a}_{1}, a_{2}, \bar{a}_{2}, \cdots, a_{n}, \bar{a}_{n}\right)=0,
$$

where $F$ is a real function with continuous first partial derivatives and $F<0$ for interior points of $V_{n}$. It can be shown that all the points of $B_{n}$ are regular except for certain manifolds of dimension less than $2 n-1$. A proof of this fact will not be given here, but can be modeled after that outlined by Schaeffer, Schiffer, and Spencer [11] for the analogous case of mappings of the interior of the unit circle. If $z=x+i y$, it will be convenient to introduce the following two operators 


$$
\frac{\partial}{\partial z}=\frac{1}{2}\left(\frac{\partial}{\partial x}-i \frac{\partial}{\partial y}\right), \quad \frac{\partial}{\partial \bar{z}}=\frac{1}{2}\left(\frac{\partial}{\partial x}+i \frac{\partial}{\partial y}\right) .
$$

We shall define

$$
F_{k}=\frac{\partial F}{\partial a_{k}}, \quad \bar{F}_{k}=\frac{\partial F}{\partial \bar{a}_{k}} .
$$

If we now make the variation (47) on functions $f_{0}(z)$ which correspond to $p_{n}$ on $B_{n}$, we obtain a new point $p_{n}^{*}=\left(a_{1}^{*}, a_{2}^{*}, \cdots, a_{n}^{*}\right)$ of $V_{n}$. The first order change in $F$ will be

$$
\delta F=\sum_{k=1}^{n}\left(F_{k} \delta a_{k}+\bar{F}_{k} \delta \bar{a}_{k}\right)=2 \operatorname{Re} \sum_{k=1}^{n} F_{k} \delta a_{k},
$$

where $\delta a_{k}$ is given by (37). Since the constant $a$ in (37) can have arbitrary argument, while $\delta F$ can never be positive, we conclude that for $|z|>1$,

$$
\begin{aligned}
\sum_{k=1}^{n} F_{k}\left\{\frac{1}{2}(k+1) a_{k}\right. & \left.+\sum_{\nu=1}^{k+1}(k-\nu) a_{k \rightarrow \nu} z^{\nu}\right\} \\
& +\bar{F}_{k}\left\{\frac{1}{2}(k+1) \bar{a}_{k}+\sum_{\nu=1}^{k+1}(k-\nu) \bar{a}_{k-\nu} z^{-\nu}\right\} \\
& =-z^{2} f_{0}^{\prime},(z)^{2} \sum_{k=1}^{n} F_{k} \frac{1}{k} R_{k}^{\prime}\left(f_{0}(z)\right) .
\end{aligned}
$$

For the function $f_{0}(z)$ corresponding to the boundary point $p_{n}$, the expression $\sum_{k=1}^{n}(k+1) F_{k} a_{k}$ is real. To prove this statement, consider the function $f_{0}^{*}(z)=e^{i \epsilon} f_{0}\left(e^{-i \epsilon} z\right)$. In this case,

$$
a_{k}^{*}=a_{k} e^{i(k+1) \epsilon}=a_{k}+i \epsilon(k+1) a_{k}+O\left(\epsilon^{2}\right),
$$

for small values of $\epsilon$. Thus $\delta F=2 \operatorname{Re} i \epsilon \sum_{k=1}^{n}(k+1) F_{k} a_{k}$, and since $\epsilon$ can either be positive or negative, while $\delta F$ can never be positive, we conclude that $\sum_{k=1}^{n}(k+1) F_{k} a_{k}$ is real.

If we set the left side of (113) equal to $\widetilde{Q}_{n}(z)$ and the multiplier of $z^{2} f_{0}^{\prime}(z)^{2}$ on the right side of (113) equal to $\widetilde{S}_{n}(z)$, we can carry out the same arguments given at the beginning of the previous chapter. We are thus able to conclude that the function $f_{0}(z)$ corresponding to a regular point of $B_{n}$ maps $C$ onto a slit domain whose boundary is composed of a finite number of analytic arcs. The function $\widetilde{Q}_{n}(z)$ is defined in the whole plane; thus we may continue the right side of (113) into $|z|<1$ and (113) holds for all $z$.

Since $f_{0}(z)$ maps $\gamma$ onto a slit, $f_{0}^{\prime}(z)$ must have at least two zeros on $\gamma$ corresponding to the tips of the slit. Let $K$ be one of these zeros; $|K|=1$. Then $\widetilde{Q}_{n}(z)$ has a double zero at $z=K$; that is, $\widetilde{Q}_{n}(K)=0$ and $K\left(d \widetilde{Q}_{n}(K) / d K\right)=0$, or 


$$
\begin{aligned}
\sum_{k=1}^{n} F_{k}\left\{\frac{1}{2}(k+1) a_{k}\right. & \left.+\sum_{\nu=1}^{k+1}(k-\nu) a_{k-\nu} K^{\nu}\right\} \\
& +\bar{F}_{k}\left\{\frac{1}{2}(k+1) \bar{a}_{k}+\sum_{\nu=1}^{k+1}(k-\nu) \bar{a}_{k-\nu} \bar{K}^{\nu}\right\}=0
\end{aligned}
$$

and

$$
\sum_{\nu=1}^{n}\left\{\sum_{\nu=1}^{k+1} \nu(k-\nu) a_{k \rightarrow \nu} F_{k} K^{\nu}-\sum_{\nu=1}^{k+1} \nu(k-\nu) \bar{a}_{k \rightarrow \nu} \bar{F}_{k} \bar{K}^{\nu}\right\}=0, \quad \bar{K}=K^{-1}
$$

The parameter $K$ may be eliminated from these two equations leaving a partial differential equation for the function $F$ representing the boundary surface $B_{n}$. The method of characteristics will now be used to construct the surface $B_{n}$ following the general plan of Schaeffer, Schiffer, and Spencer [11].

In general, let $F\left(a_{1}, \bar{a}_{1}, \cdots, a_{n}, \bar{a}_{n}\right)$ satisfy the partial differential equation

$$
\Phi\left(a_{1}, \bar{a}_{1}, \cdots, a_{n}, \bar{a}_{n}, F_{1}, \bar{F}_{1}, \cdots, F_{n}, \bar{F}_{n}\right)=0 .
$$

Then the characteristics are given in terms of a parameter $t$ by the following system of ordinary differential equations:

$$
\begin{aligned}
\frac{d a_{k}}{d t} & =2 \frac{\partial \Phi}{\partial F_{k}}, \quad \frac{d F_{k}}{d t}=-2 \frac{\partial \Phi}{\partial a_{k}}, \\
\frac{d F}{d t} & =2 \sum_{k=1}^{n}\left(\frac{\partial \Phi}{\partial F_{k}} F_{k}+\frac{\partial \Phi}{\partial \bar{F}_{k}} \bar{F}_{k}\right), \quad k=1,2, \cdots, n,
\end{aligned}
$$

the last equation being the strip condition. In our problem, the equation (115), $\widetilde{Q}\left(a_{k}, F_{k}, K\right)=0$, depends upon the $a_{k}$ and $F_{k}$ directly and also indirectly through $K$. This leads to the following system of equations for the characteristics:

$$
\begin{aligned}
& \frac{d a_{k}}{d t}=2\left(\frac{\partial \tilde{Q}}{\partial F_{k}}+\frac{\partial \tilde{Q}}{\partial K} \frac{\partial K}{\partial F_{k}}\right), \quad \frac{\partial F_{k}}{\partial t}=-2\left(\frac{\partial \tilde{Q}}{\partial a_{k}}+\frac{\partial \tilde{Q}}{\partial K} \frac{\partial K}{\partial a_{k}}\right), \\
& \frac{d F}{d t}=\sum_{\nu=1}^{n} \frac{\partial \widetilde{Q}}{\partial F_{\nu}} F_{\nu}+\frac{\partial \widetilde{Q}}{\partial K} \frac{\partial K}{\partial F_{\nu}} F_{\nu}+\frac{\partial \widetilde{Q}}{\partial \bar{F}_{\nu}} \bar{F}_{\nu}+\frac{\partial \widetilde{Q}}{\partial K} \frac{\partial K}{\partial \bar{F}_{\nu}} \bar{F}_{\nu}, \quad k=1, \cdots, n .
\end{aligned}
$$

(116), however, says that $\partial \widetilde{Q} / \partial K=0$, leaving us with the system of equations:

$$
\begin{aligned}
& \frac{d a_{k}}{d t}=(k+1) a_{k}+2 \sum_{\nu=1}^{k+1}(k-\nu) a_{k-\nu} K^{\nu}, \\
& \frac{d F_{k}}{d t}=-(k+1) F_{k}-2 k \sum_{\nu=1}^{n-k} F_{\nu+k} K^{\nu},
\end{aligned}
$$

and the strip condition 


$$
d F / d t=2 \widetilde{Q}\left(a_{k}, F_{k}, K\right) .
$$

The function corresponding to the point $p$ on $B_{n}$ maps $\gamma$ onto a curve $\Gamma$ which is composed of a finite number of analytic arcs, each satisfying the equation (analogous to (59)):

$$
\sum_{k=1}^{n} F_{k} \frac{1}{k} R_{k}^{\prime}(w)\left(\frac{d w}{d \tau}\right)^{2}=1,
$$

where $\tau$ is a real parameter on $\Gamma_{\nu}$, and $R_{k}^{\prime}(w)$ is given in (38). Using the fact that $R_{k}(w)$ is the polynomial part of $\phi_{0}(w)^{k}$, where $\phi_{0}$ is the inverse of $f_{0}$, it is easy to verify that

$$
\frac{1}{k} R_{k}^{\prime}(w)=\sum_{\nu=1}^{k} a_{k}^{(\nu)} w^{\nu \rightarrow 1}
$$

where the $a_{m}^{(\nu)}$ are determined by

$$
f_{0}(z)^{\rightarrow \nu}=\sum_{m=0}^{\infty} a_{\nu+m}^{(\nu)} z^{-\nu-m}, \quad a_{\nu}^{(\nu)}=1, \quad a_{\nu+1}^{(\nu)}=-\nu c .
$$

We may then write (123) in the form

$$
\sum_{\nu=1}^{n} A_{\nu} w^{\nu-1}\left(\frac{d w}{d \tau}\right)^{2}=1, \quad A_{\nu}=\sum_{k=\nu}^{n} F_{k} a_{k}^{(\nu)} .
$$

Our aim now is to characterize those functions $f_{0}(z, t)$ which correspond to points $p(t)$ along a given characteristic. To do so, we shall study the dependence of the coefficients $A_{\nu}$ upon the parameter $t$ of a characteristic. With this in mind, let us study the coefficient body $V_{n}^{(\nu)}$ generated by $\left(a_{\nu+2}^{(\nu)}, \cdots\right.$, $\left.a_{\nu+n+1}^{(\nu)}\right)$ for all functions $f_{0}(z)^{-\nu}$ with $f_{0}$ in $T_{0}$. Let $F^{(\nu)}\left(a_{\nu+2}^{(\nu)}, \bar{a}_{\nu+2}^{(\nu)}, \cdots, a_{\nu+n+1}^{(\nu)}\right.$, $\left.\bar{a}_{\nu+n+1}^{\nu}\right)=0$ represent a regular portion of the boundary, $B_{n}^{(\nu)}$ of $V_{n}^{(\nu)}$. For $\nu=-1$, this reduces to the problem considered above with $a_{m}^{(-1)}=a_{m}$.

The variation formula for $f_{0}(z)^{-\nu}$ can be obtained from (48); that is,

$$
\begin{array}{r}
f_{0}^{*}(x)^{-\nu}=f_{0}(x)^{-\nu}-\frac{a \rho^{2}}{2}\left[\frac{2 \nu f_{0}(x)^{-\nu}}{f_{0}(z)\left(f_{0}(x)-f_{0}(z)\right)}+\frac{z+x}{z-x} \frac{x \nu f_{0}(x)^{-\nu-1} f_{0}^{\prime}(x)}{z^{2} f_{0}^{\prime}(z)^{2}}\right. \\
\left.+\frac{\nu f_{0}(x)^{-\nu}}{z^{2} f_{0}^{\prime}(z)^{2}}\right]-\frac{\bar{a} \rho^{2}}{2}\left[\frac{1+x \bar{z}}{1-x \bar{z}} \frac{x \nu f_{0}(x)^{-\nu-1} f_{0}(x)}{\bar{z}^{2} \bar{f}_{0}^{\prime}(z)^{2}}+\frac{\nu f_{0}(x)^{-\nu}}{\bar{z}^{2} \bar{f}_{0}^{\prime}(z)^{2}}\right]
\end{array}
$$

for all $z$ in $C$. Let us define the generalized Faber polynomial in the following manner:

$$
\frac{f_{0}(x)^{-\nu}}{t\left(f_{0}(x)-t\right)}=\sum_{k=\nu+2}^{\infty} \frac{1}{k} R_{k}^{(\nu) \prime}(t) x^{-k}
$$

To obtain the variation formula for the coefficients $a_{m}^{(\nu)}$, expand (126) in 
powers of $x$ and compare coefficients; this procedure leads to

$$
\begin{aligned}
\delta a_{k}^{(\nu)}= & a_{\rho}^{2}\left[-\frac{2 \nu}{k} R_{k}^{(\nu) \prime}\left(f_{0}(z)\right)\right. \\
& \left.+\frac{1}{z^{2} f_{0}^{\prime}(z)^{2}}\left\{2 \sum_{\mu=0}^{k-\nu-1}(\nu+\mu) a_{\nu+\mu}^{(\nu)} z^{k-\nu-\mu}+(k-\nu) a_{k}^{(\nu)}\right\}\right] \\
& +\bar{a} \rho^{2}\left[\frac{1}{\bar{z}^{2} \bar{f}_{0}^{\prime}(z)^{2}}\left\{2 \sum_{\mu=0}^{k-\nu-1}(\nu+\mu) a_{\nu+\mu}^{(\nu)} \bar{z}^{-(k-\nu-\mu)}+(k-\nu) a_{k}^{(\nu)}\right\}\right] .
\end{aligned}
$$

The variation formula for $F^{(v)}$ corresponding to (112) can be constructed, giving us the differential equation (the same as (113) when $\nu=-1$ ):

$$
\begin{aligned}
\sum_{k=\nu+2}^{\nu+n+1} F_{k}^{(\nu)}\left\{\frac{1}{2}(\right. & \left.k-\nu) a_{k}^{(\nu)}+\sum_{\mu=0}^{k-\nu-1}(\nu+\mu) a_{\nu+\mu}^{(\nu)} z^{k-\nu-\mu}\right\} \\
+ & \bar{F}_{k}^{(\nu)}\left\{\frac{1}{2}(k-\nu) \bar{a}_{k}^{(\nu)}+\sum_{\mu=0}^{k-\nu-1}(\nu+\mu) \bar{a}_{\nu+\mu}^{(\nu)} z^{-(k-\nu-\mu)}\right\} \\
= & z^{2} f_{0}^{\prime}(z)^{2} \sum_{k=\nu+2}^{\nu+n+1} F_{k}^{(\nu)} \frac{\nu}{k} R_{k}^{(\nu)}\left(f_{0}(z)\right), \quad F_{k}^{(\nu)}=\partial F^{(\nu)} / \partial a_{k}^{(\nu)} .
\end{aligned}
$$

Again setting $Q_{n}^{(\nu)}(z)$ equal to the left side of (129) and $z^{2} f_{0}^{\prime}(z)^{2} S_{n}^{(\nu)}\left(f_{0}(z)\right)$ equal to the right side, we prove as before that $Q_{n}^{(\nu)}(z)$ is non-negative for $z$ on $\gamma$, and that the image $\Gamma$ of $\gamma$ under mappings $f_{0}$ that correspond to boundary points $p^{(v)}$ of $B_{n}^{(\nu)}$ will be a slit composed of analytic $\operatorname{arcs} \Gamma_{i}$, each satisfying the differential equation

$$
S_{n}^{(\nu)}(w(\tau))(d w / d \tau)^{2}+1=0,
$$

for some real parameter $\tau$.

If we let $K$ be one of the points on $\gamma$ which is mapped into the tip of the slit $\Gamma, f_{0}^{\prime}(K)=0$; and we are led to the differential equation

$$
\begin{aligned}
\sum_{k=\nu+2}^{\nu+n+1} F_{k}^{(\nu)}\left\{\frac{1}{2}(k\right. & \left.-\nu) a_{k}^{(\nu)}+\sum_{\mu=0}^{k-\nu-1}(\nu+\mu) a_{\nu+\mu}^{(\nu)} K^{k-\nu-\mu}\right\} \\
& +\bar{F}_{k}^{(\nu)}\left\{\frac{1}{2}(k-\nu) a_{k}^{(\nu)}+\sum_{\mu=0}^{k-\nu-1}(\nu+\mu) \bar{a}_{\nu+\mu}^{(\nu)} \bar{K}^{k-\nu-\mu}\right\}=0
\end{aligned}
$$

where $K$ is eliminated by the condition $K\left(d Q_{n}^{(\nu)}(K) / d K\right)=0$; that is,

$$
\begin{aligned}
\sum_{k=\nu+2}^{\nu+n+1}\left\{F_{k}^{(\nu)} \sum_{\mu=0}^{k-\nu-1}(\nu\right. & +\mu)(k-\nu-\mu) a_{\nu+\mu}^{(\nu)} K^{k-\nu-\mu} \\
& \left.\quad \bar{F}_{k}^{(\nu)} \sum_{u=0}^{k-\nu-1}(\nu+\mu)(k-\nu-\mu) \bar{a}_{\nu+\mu}^{(\nu)} \bar{K}^{k-\nu-\mu}\right\}=0 .
\end{aligned}
$$


The characteristic equations for the differential equation (130) are

(132) $\frac{d a_{k}^{(\nu)}}{d t}=(k-\nu) a_{k}^{(\nu)}+2 \sum_{\mu=1}^{k-\nu}(k-\mu) a_{k-\mu}^{(\nu)} K^{\mu}$,

(133) $\frac{d F_{k}^{(\nu)}}{d t}=-(k-\nu) F_{k}^{(\nu)}-2 k \sum_{\mu=1}^{\nu+n+1-k} F_{\mu+k}^{(\nu)} K^{\mu}, k=\nu+2, \cdots, \nu+n+1$,

and the strip condition

$$
\frac{d F^{(\nu)}}{d t}=2 Q_{n}^{(\nu)}(K)
$$

Let us define

$$
\begin{array}{lll}
\text { (135) } \alpha_{k}^{(\nu)}=e^{(\nu+1) t} a_{k}^{(\nu)} & \text { for } k \geqq \nu, & \alpha_{k}^{(\nu)}=0 \text { for } k<\nu, \\
\text { (136) } \phi_{k}^{(\nu)}=e^{-(\nu+1) t} F_{k}^{(\nu)} \text { for } k \leqq \nu+n+1, & \phi_{k}^{(\nu)}=0 \text { for } k>\nu+n+1 .
\end{array}
$$

Then the equations (132) and (133) of the characteristics become

(137) $\frac{d \alpha_{k}^{(\nu)}}{d t}=(k+1) \alpha_{k}^{(\nu)}+2 \sum_{\mu=1}^{k+1}(k-\mu) \alpha_{k-\mu}^{(\nu)} K^{\mu}$,

(138) $\frac{d \phi_{k}^{(\nu)}}{d t}=-(k+1) \phi_{k}^{(\nu)}-2 k \sum_{\mu=1}^{n} \phi_{\mu+k}^{(\nu)} K^{\mu}, \quad k=\nu+2, \cdots, \nu+n+1$.

Thus $\alpha_{k}^{(\nu)}$ and $\phi_{k}^{(\nu)}$ satisfy the same characteristic equation for all values of $\nu$.

Returning to (125), we see that

$$
A_{\nu} e^{(\nu+1) t}=\sum_{k=\nu}^{n} \alpha_{k}^{(\nu)} \phi_{k}^{(-1)}
$$

For $\nu=1,2, \cdots, n$, however, we may apply (137) and (138) to obtain

$$
\begin{aligned}
\frac{d}{d t} \sum_{k=\nu}^{n} \alpha_{k}^{(\nu)} \phi_{k}^{(-1)}= & 2 \sum_{k=\nu}^{n}\left\{\sum_{\mu=1}^{k+1}(k-\mu) \alpha_{k-\mu}^{(\nu)} \phi_{k}^{(-1)} K^{\mu}\right. \\
& \left.-\sum_{\mu=1}^{n} k \phi_{\mu+k}^{(-1)} \alpha_{k}^{(\nu)} K^{\mu}\right\} .
\end{aligned}
$$

Since $\alpha_{k}^{(\nu)}=0$ for $k<\nu$, we can extend the summation over $k$ in (140) from 1 to $n$. Reversing the order of summation in (140), we find that

$$
\begin{aligned}
\frac{d}{d t} \sum_{k=\nu}^{n} \alpha_{k}^{(\nu)} \phi_{k}^{(-1)} & =2 \sum_{\mu=1}^{n}\left\{\sum_{k=\mu+1}^{n}(k-\mu) \alpha_{k-\mu}^{(\nu)} \phi_{k}^{(-1)}-\sum_{k=1}^{n} k \phi_{k+\mu}^{(-1)} \alpha_{k}^{(\nu)}\right\} K^{\mu} \\
& =2 \sum_{\mu=1}^{n}\left\{\sum_{k=-1}^{n+\mu} k \alpha_{k}^{(\nu)} \phi_{k+\mu}^{(-1)}-\sum_{k=1}^{n} k \phi_{k+\mu}^{(-1)} \alpha_{k}^{(v)}\right\} K^{\mu} .
\end{aligned}
$$


Since $\phi_{k+\mu}^{(-1)}=0$ for $n-\mu<k \leqq n$, and $\alpha_{-1}^{(\nu)}=\alpha_{0}^{(\nu)}=0$ for $\nu=1,2, \cdots, n$, the two sums in the brackets in (141) are identical, leaving us with $d \sum_{k=\nu}^{n} \alpha_{k}^{(\nu)} \phi_{k}^{-1} / d t$ $=0$. Therefore

$$
A_{\nu}=c_{\nu} e^{-(\nu+1) t}
$$

where the $c_{\nu}$ are constants.

If we now set $w=e^{t} \omega$ in (125), we get

$$
\sum_{\nu=1}^{n} c_{\nu} \omega^{\nu-1}\left(\frac{d \omega}{d \tau}\right)^{2}=1
$$

which is independent of $t$. Corresponding to the function $f_{0}(z, t)$ belonging to the point $p(t)$ of the characteristic, the function

$$
\omega=g_{0}(z, t)=e^{-t} f_{0}(z, t)=e^{-t}\left(z+\frac{a_{1}}{z}+\frac{a_{2}}{z^{2}}+\cdots\right)
$$

maps $\gamma$ onto an analytic slit which satisfies equation (143) for all values of $t$ and passes through the origin.

We shall define the $\Gamma^{0}$-structure corresponding to a given characteristic as the complete solution of (143) which passes through the origin. Thus $\boldsymbol{\omega}=g_{0}(z, t)$ maps $\gamma$ onto a portion of the $\Gamma^{0}$-structure for all values of $t$. Let us fix an initial point on $B_{n}$ corresponding to $f_{0}(z, 0)$; that is, $t=0$. We then continue the characteristic in the direction of increasing $t$. The slit, which is the image of $\gamma$ under $g_{0}(z, t)$, forms a subcontinuum $\Gamma_{t}^{0}$ of the $\Gamma^{0}$-structure for this characteristic, and as $t$ increases, the factor $e^{-t}$ indicates that $\Gamma_{t}^{0}$ will shrink, covering less of $\Gamma^{0}$.

Returning now to the family $T$, we recall that $f_{0}(z, t)=f(z, t)+c(t)$, where $c(t)$ is only a function of $t$ and $f(z, t)$ is in $T$. If we define $g(z, t)=e^{-t} f(z, t)$, we see that $g(z, t)=g_{0}(z, t)-e^{-t} c(t)$. Thus $g(z, t)$ maps $\gamma$ onto a slit $\Gamma_{t}$ which is the translate of $\Gamma_{t}^{0}$ through a distance $e^{-t} c(t)$.

This idea of a shrinking slit is similar to that used by Loewner [9] in obtaining his differential equation for univalent mappings of the circle. We shall now show that along the characteristic curve on $B_{n}$, the equations $(120 \mathrm{a})$ and $(120 \mathrm{~b})$ imply that $g(z, t)$ satisfies the Loewner equation.

First it will be necessary to prove the uniqueness of the function in $T$ corresponding to a regular boundary point of $V_{n}$ by showing that for a given set of $a_{i}$ and $F_{i}$, there is a unique function in $T$ satisfying the differential equation (113) with $f_{0}$ replaced by $f$. The Faber polynomial $R_{k}(w)$ can also be defined (Schiffer [14]) as the unique polynomial of degree $k$ in $w$ such that

$$
R_{k}(f(z))=z^{k}+\frac{c_{k 1}}{z}+\frac{c_{k 2}}{z^{2}}+\cdots .
$$

It was shown by Schur [15] that 


$$
c_{k j}=b a_{k+j}+P_{1}\left(a_{1}, \cdots, a_{k+j-1}\right),
$$

where $b$ is an integer and $P_{1}$ represents a polynomial in its arguments. Since

$$
R_{k:}^{\prime}(w)=\frac{1}{f^{\prime}(z)} \frac{d}{d z} R_{k}(f(z)), \quad w=f(z),
$$

we can write the right side of (113) as

$$
\sum_{k=1}^{n} \frac{1}{k} F_{k} z^{2} f^{\prime}(z) \frac{d}{d z} R_{k}(f(z)) .
$$

Introducing the expansion (144) into (147), we obtain

$$
\sum_{k=1}^{n} \frac{1}{k} F_{k} \cdot\left(z-\frac{a_{1}}{z}-\frac{2 a_{2}}{z^{2}}-\cdots\right)\left(k z^{k}-\frac{c_{k 1}}{z}-\frac{2 c_{k 2}}{z^{2}}-\cdots\right) .
$$

In view of (145) and (148), comparison of the coefficients of $z^{-i}$ on the two sides of (3.5) yields a relation of the form

$$
a_{n+i+1}=P_{2}\left(a_{1}, \cdots, a_{n+i}\right),
$$

where again $P_{2}$ is used to represent a polynomial in its arguments. The relation (149) between the coefficients of $f(z)$ can be used as a recursion formula for $a_{m}, m>n$. Thus if the coefficients $a_{1}, a_{2}, \cdots, a_{n}$ and the numbers $F_{1}, F_{2}, \cdots, F_{n}$ are given, the function $f(z)$ is completely determined by the recursion formulas.

Let us now consider the boundary point $p(t):\left(a_{1}(t), a_{2}(t), \cdots, a_{n}(t)\right)$ on the characteristic $\chi$ corresponding to the function $f(z, t)$. At this point the form $\left(F_{1} a_{1}+\bar{F}_{1} \bar{a}_{1}+\cdots+F_{n} a_{n}+\bar{F}_{n} \bar{a}_{n}\right)$ assumes a relative maximum for $f$ in $T$, the $F_{1}, \bar{F}_{1}, \cdots, F_{n}, \bar{F}_{n}$ giving the (complex) direction numbers of the normal to $B_{n}$ at $p(t)$. If we now consider the surface $V_{n+\mu}$ (again assumed to be regular) generated by points $\left(a_{1}, \cdots, a_{n+\mu}\right)$ for $f$ in $T$, we can find a point $\tilde{p}(t)$ on the surface $B_{n+\mu}$ where the direction numbers of the normal are $F_{1}, \bar{F}_{1}, \cdots, F_{n}, \bar{F}_{n}, 0, \cdots, 0$. This is true since the tangent plane at a regular point $\tilde{p}(t)$ which projects into a boundary point of $V_{n}$ must be orthogonal to the subspace of $2(n+\mu)$-dimensional space generated by $a_{1}, a_{2}, \cdots, a_{n}$. For otherwise, considering the tangent plane as an approximation to the surface $B_{n+\mu}$, we see that $\tilde{p}(t)$ would project into a point $p$ of $V_{n}$ having a complete neighborhood about it in $V_{n}$ and hence could not be a boundary point of $V_{n}$.

The function $\tilde{f}(z, t)$ corresponding to the point $\tilde{p}(t)$ is the same as the function $f(z, t)$ corresponding to $p(t)$. For $\left(a_{1}(t), a_{2}(t), \cdots, a_{n}(t)\right)$ also gives a relative maximum to the form $F_{1} a_{1}+\bar{F}_{1} \bar{a}_{1}+\cdots+F_{n+\mu} a_{n+\mu}+\bar{F}_{n+\mu} \bar{a}_{n+\mu}$ in which $F_{n+\nu}=0$ for $\nu=1, \cdots, \mu$. Then the function $\tilde{f}(z, t)$ satisfies equation (113) for the same values of $a_{\nu}$ and $F_{\nu}$ as does $f(z, t)$; hence by the uniqueness proved above, $\tilde{f}(z, t)=f(z, t)$. 
We observe that in (120b), $d F_{k} / d t$ is expressed in terms of $F_{k+\nu}, \nu>0$, and thus are all zero at $\tilde{p}(t)$ for $k>n$. For $k=1, \cdots, n$, the equations of the characteristic on $V_{n+\mu}$ passing through $\tilde{p}(t)$ are exactly the same as those given in (120a and $120 \mathrm{~b})$ for the characteristic $\chi$ through $p(t)$, since the $d a_{k} / d t$ depend only upon the $a_{\nu}$ for $\nu \leqq k, k=1,2, \cdots, n$. Furthermore (120a) is also satisfied for $k=n+1, \cdots, n+\mu$ and at $\tilde{p}(t), d F_{k} / d t=0$ for $k>n$. But the first $n$ equations in (120a and $120 \mathrm{~b})$ are exactly the equations satisfied by the coefficients of $f(z, t)$ as we move along the characteristic $\chi$ of $V_{n}$, so we see that $f(z, t)$ also corresponds to a single characteristic $\tilde{\chi}$ on $V_{n+\mu}$. Therefore, the first $n+\mu$ coefficients of $f(z, t)$ satisfy (120a) and since $\mu$ is arbitrary, we can say that all the coefficients of $f(z, t)$ satisfy $(120 \mathrm{a})$.

Expanding the following two expressions in powers of $z$, we obtain

$$
\begin{aligned}
z \frac{\partial g(z, t)}{\partial z} \frac{z+K}{z-K} & =e^{-t} z-e^{-t} \sum_{\nu=2}^{\infty}\left(\nu a_{\nu}+2 \sum_{k=1}^{\nu+1}(\nu-k) a_{\nu-k} K^{k}\right) z^{-\nu} \\
\frac{\partial g(z, t)}{\partial t} & =-e^{-t} z+e^{-t} \sum_{\nu=1}^{\infty}\left(-a_{\nu}+\frac{d a_{\nu}}{d t}\right) z^{-\nu}
\end{aligned}
$$

If we substitute the value of $d a_{v} / d t$ along the characteristic given by (120a), we see that

$$
\frac{\partial g(z, t)}{\partial t}=-z \frac{\partial g(z, t)}{\partial z} \frac{z+K}{z-K}
$$

where $K=K(t)$ is a point on $\gamma$ which corresponds to a tip of the image $\Gamma_{t}$. The differential equation (152) is the Loewner differential equation.

\section{BIBLIOGRAPHY}

1. L. Bieberbach, Ueber die Koeffizienten derjenigen Potenzreihen, welche eine schlichte Abbildung des Einheitskreises vermitteln, Preuss. Akad. Wiss. Sitzungsber. (1916) pp. 940-955.

2. M. Biernacki, Sur quelques majorantes de la théorie des fonctions univalentes, C. R. Acad. Sci. Paris vol. 201 (1935) pp. 256-258.

3. G. Faber, Neuer Beweis eines Koebe-Bieberbachschen Satzes ueber konforme Abbildung, Akademie der Wissenschaften, Munich, Sitzungsberichte der Mathematisch-physische Klasse (1918) pp. 39-42.

4. P. R. Garabedian, and M. Schiffer, Identities in the theory of conformal mapping, Trans. Amer. Math. Soc. vol. 65 (1949) pp. 187-238.

5. G. M. Golusin, Einige Koeffizientenabschaetzungen fuer schlicht Funktionen, Rec. Math. (Mat. Sbornik) N.S. vol. 3 (1938) pp. 321-330.

6. T. H. Gronwall, Some remarks on conformal representation, Ann. of Math. vol. 16 (19141915) pp. 72-76.

7. J. Hadamard, Mémoire sur le problème d'analyse relatif a l'équilibre des plaques elastiques encastrêes, Mémoires de l'Académie des Sciences de l'Institut de France vol. 38 (1908).

8. G. Julia, Sur une équation aux derivées fonctionelles, Ann. Ecole Norm. (3) vol. 39 (1922) pp. 1-28.

9. K. Loewner, Untersuchungen ueber schlichte konforme Abbildungen des Einheitskreises, Math. Ann. vol. 89 (1923) pp. 103-121. 
10. F. Marty, Sur le module des coefficients de MacLaurin d'une fonction univalente, C. R. Acad. Sci. Paris vol. 198 (1934) pp. 1569-1571.

11. A. C. Schaeffer, M. Schiffer, and D. C. Spencer, The coefficient regions of schlicht func. tions, Duke Math. J. vol. 16 (1949) pp. 493-527.

12. M. Schiffer, $A$ method of variation within the family of simple functions, Proc. London Math. Soc. (2) vol. 44 (1938) pp. 432-449.

13. ——, Sur un problème d'extrémum de la représentation conforme, Bull. Soc. Math. France vol. 66 (1938) pp. 48-55.

14. - Faber polynomials in the theory of univalent functions, Bull. Amer. Math. Soc. vol. 54 (1948) pp. 503-517.

15. I. Schur, On Faber polynomials, Amer. J. Math. vol. 67 (1945) pp. 33-41.

16. O. Teichmueller, Ungleichungen zwischen den Koeffizienten schlichter Funktionen, Preuss. Akad Wiss. Sitzungsber. (1938) pp. 363-375.

17. J. L. Walsh, Interpolation and approximation, Amer. Math. Soc. Colloquium Publications, vol. 20, New York, 1935, pp. 68-71.

Massachusetts Institute of Technology,

Cambridge, Mass. 